\title{
Node-covering, Error-correcting Codes and Multiprocessors with Very High Average Fault Tolerance
}

\author{
Shantanu Dutt, Member, IEEE, and Nihar R. Mahapatra, Member, IEEE
}

\begin{abstract}
Structural fault tolerance (SFT) is the ability of a multiprocessor to reconfigure around faulty processors or links in order to preserve its original processor interconnection structure. In this paper, we focus on the design of SFT multiprocessors that have low switch and link overheads, but can tolerate a very large number of processor faults on the average. Most previous work has concentrated on deterministic $k$-fault-tolerant $(k-\mathrm{FT})$ designs in which exactly $k$ spare processors and some spare switches and links are added to construct multiprocessors that can tolerate any $k$ processor faults. However, after $k$ faults are reconfigured around, much of the extra links and switches can remain unutilized. It is possible within the basic node-covering framework, which was introduced by Dutt and Hayes as an efficient $k$-FT design method, to design FT multiprocessors that have the same amount of switches and links as, say, a two-FT deterministic design, but have $s$ spare processors, where $s \gg 2$, so that, on the average, $k=\Theta(s)(k \leq s)$ processor failures can be reconfigured around. Such designs utilize the spare link and switch capacity very efficiently, and are called probabilistic FT designs. An elegant and powerful method to construct covering graphs or CG's, which are key to obtaining the probabilistic FT designs, is to use linear error-correcting codes (ECCs). We show how to construct probabilistic designs with very high average fault tolerance but low wiring and switch overhead using ECCs like the 2D-parity, full-two, 3D-parity, and full-three codes. This design methodology is applicable to any multiprocessor interconnection topology and the resulting FT designs have the same node degree as the non-FT target topology. We also analyze the deterministic fault tolerance for these designs and develop efficient layout strategies for them. Finally, we compare the proposed probabilistic designs to some of the best deterministic and probabilistic designs proposed in the past, and show that our designs can meet a given mean-time-to-failure (MTTF) specification at much lower hardware costs (switch complexity, redundant wiring area, and spare-processor overhead) than previous designs. Further, for a given number of spare processors, our designs have close-to-optimal reconfigurabilities that are much better than those of previous probabilistic designs.
\end{abstract}

Index Terms-Average fault tolerance, deterministic fault tolerance, fault-tolerant multiprocessors, linear error-correcting codes, matching, network flow, node-covering, VLSI layout, reconfiguration.

\section{INTRODUCTION AND MOTIVATION} $\mathrm{T}$ is important to design multiprocessors with fault toler1 ance and reconfiguration capabilities so that they continue to function correctly and with very little degradation, in spite of a few processor/link failures, and without requiring the applications to be coded for fault tolerance. To achieve this, multiprocessors need to be designed with structural fault tolerance (SFT), which is the ability to reconfigure around faulty components (processors or links) in order to preserve the original processor interconnection structure. SFT is also useful in maintaining correct and nondegraded operation of specialized processor arrays like those used for signal and image-processing [21]. The SFT (or FT, for brevity) design methodologies that we will develop can thus be applied to both general-purpose multi-

- S. Dutt is with the Department of Electrical Engineering and Computer Science, University of Illinois at Chicago, 1120 Science and Engineering Offices, 851 S. Morgan St. Chicago, IL 60607-7053. E-mail:dutt@eecs.uic.edu.

- N.R. Mahapatra is with the Department of Electrical and Computer Engineering, State University of New York at Buffalo, Buffalo, NY 14260. E-mail:mahapatr@eng.buffalo.edu.

Manuscript received 5 May 1995; revised 8 Dec. 1995.

For information on obtaining reprints of this article, please send e-mail to: tc@computer.org, and reference IEEECS Log Number 105216. processors and special-purpose VLSI processor arrays for signal/image-processing applications.

A number of researchers have proposed various design methods for structural fault tolerance in multiprocessors, for example, [2], [4], [5], [6], [7], [8], [10], [11], [12], [13], [14], [15], [19], [22], [23], [24], [25], [26], [27], [28]. Most of this work has dealt with the design of deterministic $k$-faulttolerant $(k-\mathrm{FT})$ multiprocessors in which there are $k$ or more spare processors, and the link and switch complexity is high enough to tolerate any $k$ processor failures (but not more than $k$ processor failures) [2], [4], [5], [6], [7], [8], [10], [11], [12], [13], [14], [15], [19], [27], [23], [24]. Unfortunately, in such designs, around 50 percent of the redundant link/switch overhead remains unutilized, on the average, after $k$ faulty processors have been reconfigured around. It intuitively seems possible to utilize this remaining spare link and switch capacity of the system by putting in $s>k$ spare processors so that, on the average, more than $k$ faults can be reconfigured around. Looking at this from a different angle, we would like to design FT multiprocessors in which the link/switch complexity is kept quite low (for example, corresponding to that of a two-FT deterministic design), but many more $(s \gg 2)$ spare processors are added so that $k$ processor faults can be reconfigured around, on 


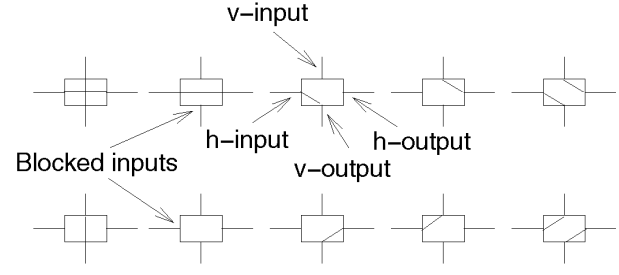

(a)

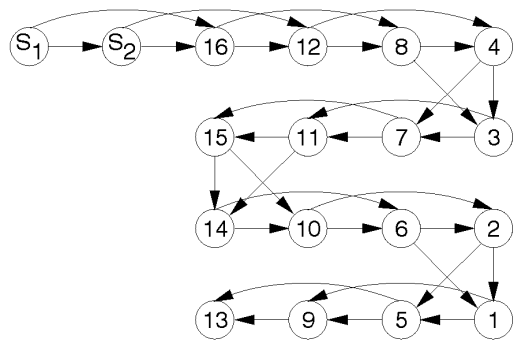

(b)

Fig. 1. (a) Possible settings of a recon-switch. (b) A standard CG for a two-FT 16-processor system of arbitrary topology arranged on a twodimensional layout-the linear ordering $\mathcal{L}$ corresponds to a snake-like row-major ordering of this $2 \mathrm{D}$ layout.

the average (or with very high probability), where $k<s$ and $k \gg 2$. This will also lead to very high utilization of the spare link/switch capacity. Since the hardware complexities in almost all multiprocessor systems are wire dominated, it is important to keep the link redundancy as low as possible in an FT design; the processor complexity is not that critical, and, thus, adding some excess, say, 5-10 percent, processor redundancy does not significantly affect the hardware complexity of the system. Since such designs are geared toward having a large fault tolerance on the average (over all fault patterns), but the worst-case fault tolerance may be lower, we call them probabilistic FT designs. Such probabilistic designs have also been proposed previously, though mainly for 2D mesh-connected multiprocessors [6], [22], [26], [27], [28]. In contrast, in this paper, we develop probabilistic designs that are applicable to any topology, and, for the special case of the 2D mesh, we compare our resulting designs' reconfigurabilities and hardware overheads to those of the best previous probabilistic designs [6], [27], [28].

In earlier work, Dutt and Hayes developed a very efficient methodology called node-covering for designing deterministic $k$-FT multiprocessors [11], [14], [15]. The basic node-covering technique will be the underlying design process for probabilistic FT, with linear codes being used to determine the interconnection between the external switches used for reconfiguration in order to obtain very high average FT.

Before proceeding further, we first define average fault tolerance as

$k_{\text {avg }}=\sum_{\mathrm{i}=1}^{\mathrm{s}}$ (Probability of tolerating a size- $i$ fault that is not

contained in a tolerable fault pattern of a larger size) $\cdot i$

A tolerable $j$-fault pattern will contain $i$-fault patterns, where $j>i$, that should not be counted separately as tolerable $i$-fault patterns. Assuming that tolerable $i$-fault patterns are uniformly distributed over the space of tolerable and intolerable fault patterns of larger sizes and that $p_{i}$ is the probability of tolerating any $i$-fault pattern (this, we might determine by, say, simulation), we have

$$
k_{\text {avg }}=\sum_{i=1}^{s}\left(p_{i} \prod_{j=i+1}^{s}\left(1-p_{j}\right)\right) \cdot i
$$

Note that, for a $k$-FT system, $k_{\text {avg }}=k$.

In Section 2, we briefly describe the node-covering method for $k$-FT designs and set the stage for discussing the probabilistic design method. In Section 3, we begin the description of the probabilistic method by discussing the concept of partitioning a multiprocessor into intersecting FT processor groups. In the next section, we give the rationale for using error-correcting codes (ECCs) as the basis for determining such processor groupings. Section 5 describes efficient layouts of some ECC-based FT designs and obtains the area overheads in each case. Next, Section 6 describes how we model and solve the reconfiguration process in our FT designs as a network-flow problem. In Section 7, we determine the deterministic fault tolerance (DFT), which is the maximum number of faults that are guaranteed to be tolerated, of each ECC-based design. Section 8 presents comparisons of reconfigurabilities and hardware overheads of ECC-based FT designs with previous designs. Finally, we conclude in Section 9. Due to space constraints, we give some of our results without proofs, which can be found in [16].

\section{The Deterministic Node-Covering Method}

In this technique [11], [14], we designate a small subset $\operatorname{cov}(v)$ of nodes (i.e., processors) of the FT multiprocessor $G^{\prime}$ to which $v$ can be mapped (i.e., by which $v$ can be replaced) for reconfiguring around faults. The set $\operatorname{cov}(v)$ is called the cover set of $v$, while each node $u$ in $\operatorname{cov}(v)$ is called a cover of $v$, denoted by $u>v$. The node $v$ is said to be a dependent of all its covers, and the set of dependents of a node $u$ is denoted by $\operatorname{dep}(u)$. Note that, for SFT, we need some mechanism for each node $u$ to be connected to the neighbors of a dependent node $v$ in the event it replaces $v$; we will shortly describe this mechanism.

A covering graph (CG) of an FT multiprocessor $G^{\prime}$ is a directed graph, whose node set consists of all the nodes of $G^{\prime}$ (including the spare nodes), and whose arc set is $\{(u, v): v \in$ $V(G), u \in \operatorname{cov}(v)\}$, where $V(G)$ is the node set of the non-FT multiprocessor $G$; see Fig. 1b. A covering sequence for a node $v$ in the CG is an ordered subset $\left(v, v_{1}, v_{2}, \ldots, v_{h}\right)$ of the node set of the CG, such that $v_{1} \succ v, v_{j}>v_{j-1}$, for $1<j \leq h$, and $v_{h}$ is the only spare node in this ordered set. Basically, a covering sequence for $v$ specifies a mapping which can be performed when $v$ is faulty, so that $v$ is replaced by $v_{1}$, which, in turn, is replaced by $v_{2}$, and so on, until $v_{h-1}$ is replaced by the spare node $v_{h}$, thus configuring it into the system. In order to be able to reconfigure around any $k$ faults, we need a set of $k$ nodedisjoint covering sequences, one for each fault, in the CG.

In order to provide each node with the necessary switching capability so that it can connect to the neighbors 


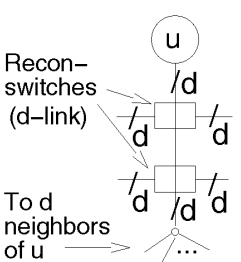

(a)

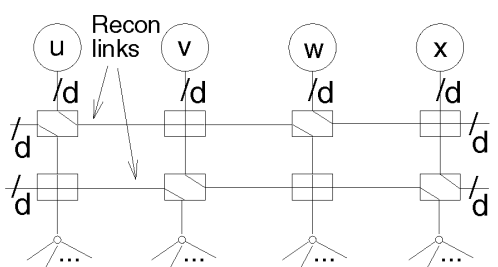

(b)
Fig. 2. (a) Connecting $u$ to its neightbors via its recon-switches. (b) An interconnection between recon-switches of four consecutive nodes in $\mathcal{L}$ for a two-FT design, and the switch settings for allowing $u$ to replace $w$ and $v$ to replace $x$.

of any dependent node that it replaces, we use a $2 \times 2$ switch, called a recon-switch, in our designs, whose possible states are specified in Fig. 1a. This switch has four bidirectional terminals (in spite of their labels, like " $v$-input" and "h-output," these are all bidirectional) and can connect any pair of them together as shown. These terminals can have any width (number of wires), and a switch with $d$ wires at each terminal is termed a $d$-link switch. In a $k$-FT system, each processor is connected to its $d$ neighbors via the $v$ inputs and $v$-outputs of $k d$-link recon-switches, as shown in Fig. 2a for $k=2$.

For an efficient $k$-FT design, we need a streamlined way of allowing a node to replace any of its $k$ dependent nodes. This is done by determining a linear ordering $\mathcal{L}$ of the nodes and by linking the $h$-outputs of each of the $k$ reconswitches of node $u$ to the $h$-inputs of the corresponding recon-switches of the node $v$ to its immediate right in $\mathcal{L}$ by reconfiguration d-links (recon d-links or, simply, recon-links); see Fig. 2b. The underlying CG of this interconnection structure is a standard CG in which each node is covered by the $k$ nodes immediately preceding it in $\mathcal{L}$; see Fig. 1 b. Now, for node $u$ to replace a dependent, say, $w$ (either when $w$ becomes faulty or when $w$ replaces one of its dependents in the covering sequence of a faulty node), the $d$ links from $u$ are reconfigured to connect to the $h$-inputs of $w$ 's recon-switch, after passing through the recon-switch of any intermediate processor $v$, and, thereby, to $w$ 's neighbors; the links from $w$ will be blocked by its first reconswitch if it is faulty, or a similar connection will be established from $w$ to the neighbors of the node it replaces (Fig. 2b). In order to minimize the link overhead, $\mathcal{L}$ should be determined from an optimal 2D or 3D layout of the multiprocessor so that adjacent nodes in $\mathcal{L}$ are also adjacent in the layout. Then, since recon-links are only required between spatially adjacent processors, the wiring overhead of the $k$-FT design is minimized. It can be shown that, assuming uniformly distributed fault locations, about 50 percent of the spare links and switches remain unutilized after all $k$ faults have been reconfigured around in a node-covering $k$-FT design [11], [14] (see [16] for proof); this also applies to other $k$-FT designs, e.g., those in [5], [7], [8].

\section{Intersecting FT Processor Groups}

In order to obtain high fault tolerance at reduced reconswitch and recon-link overheads, we need to partition the processors into smaller groups that are individually made, say, $k_{1}-\mathrm{FT}$, using the technique described in the previous section. The rationale for partitioning into smaller FT processor groups is as follows: Assuming the model of uniformly and independently distributed processor faults, the reliability of a $k$-FT system with $N$ primary processors is

$$
R_{k}(N)=\sum_{i=0}^{k}\left(\begin{array}{c}
N+k \\
i
\end{array}\right) p^{i}(1-p)^{N+k-i}
$$

where $p$ is the failure probability of a single processor. If we partition the system into $t$ disjoint groups, and make each group $k_{1}-\mathrm{FT}$, then the system's reliability is $\left(R_{k_{1}}(N / t)\right)^{t}$. If $k_{1}=k$, then it is easy to see that the partitioned system has higher reliability at the same recon-link and switch cost (but more spare processor cost, which is not that critical). Even for $k_{1}<k$, we can get the same or higher reliability in the partitioned system at reduced link and switch overheads. For example, when $p=0.02$, the reliability $R_{2}(16)$ of a twoFT 16-processor system is 0.994 , whereas a comparable reliability of 0.984 is obtained when the system is partitioned into four disjoint groups, each of which is made only oneFT. This disjoint-group based FT design is also termed local sparing, since each spare can replace processors in only its local subsystem, and is discussed in [14].

A crucial question is whether these processor groups should be disjoint or intersecting. Fig. 3a shows a disjoint grouping of processors into two groups, each of which is made two-FT, while Fig. 3b shows a nondisjoint grouping of processors into four groups, each of which is made oneFT. Each group is shown as a thick line spanning a linear processor space. The hardware overheads of the two processor-grouping schemes are similar-each requires four spare processors, and two recon-switches and recon $d$-links per processor (in Fig. 3b, each processor belongs to two groups, and one recon-switch is used to connect it to one of its groups and the second one to the other group). It is easy to see that the intersecting-groups scheme can tolerate all fault patterns that the disjoint-groups scheme can (assume, for the moment, that each group can reconfigure around faults in it without any conflict with reconfigurations in intersecting groups-we will shortly show that this is true for our designs). Furthermore, the former scheme can tolerate many more fault patterns than the latter. For example, the three-fault pattern shown in Fig. 3 cannot be tolerated in the disjoint-grouping case, but it can be tolerated in the intersecting-groups scheme as follows: The leftmost fault can be reconfigured in Group 4, the middle one in Group 1, and the third one in Group 2. This simple example clearly shows that, everything else being equal, an intersecting processor grouping yields more fault tolerance and reliability than a disjoint processor grouping. This is essentially because intersecting processor groups have many more paths between processors and spares than disjoint groups. The next crucial question is whether there is a systematic method to form different types of intersecting processor groups that have different fault tolerance and hardware cost properties. The answer to this is in the affirmative. In particular, we can draw upon linear error-correcting codes (ECCs) in order to form processor groupings; different 


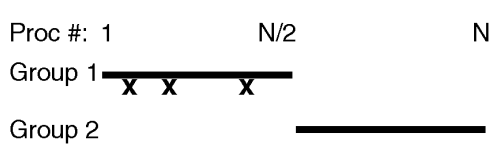

(a)
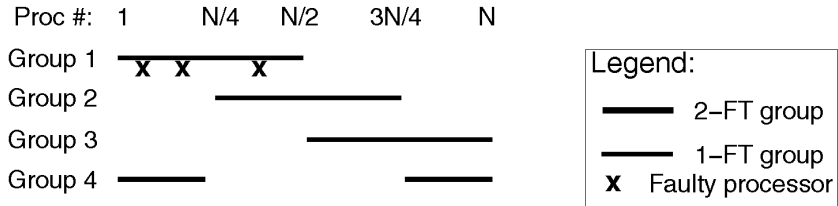

(b)

Fig. 3. (a) A disjoint processor grouping into two two-FT groups; it cannot tolerate the three-fault pattern shown. (b) An intersecting processor grouping into four one-FT groups that has a similar hardware overhead to that of the disjoint grouping; it can tolerate the three-fault pattern.

ECCs will yield FT designs with different properties with respect to the above parameters. In Section 4, we show how ECCs can be used to form (intersecting) processor groups, and how the deterministic and average error detectability of an ECC form lower bounds for the deterministic and average fault tolerance of the corresponding FT multiprocessor, and, in general, why they are a good choice to determine these groupings. Some previous probabilistic methods [22], [26], [28] have also used intersecting processor groups for the special case of the 2D mesh topology; their processor groups are the rows and columns of the 2D mesh, and, thus, they fortuitously happen to be based on the 2D-parity code. In Section 8, we compare our designs to some of the best of such previous designs.

\subsection{Switching Structure}

The switch implementation of an FT multiprocessor with intersecting processor groups is an extension of the deterministic $(k-\mathrm{FT})$ node-covering design described in Section 2. We assume, for simplicity, that each processor group is made one-FT, and, thus, has a single spare processor. Suppose each processor belongs to $d_{g}$ groups. Then, we connect $d_{g}$ recon-switches to each processor in series (via v-input and -output terminals as in $k$-FT designs). We label the recon-switches from top (to which the processor is directly connected) to bottom as $d_{g}-1, \ldots, 0$. For each processor group $P_{i}$, we order the processors in it in some manner (essentially by physical proximity, as described in Section 5), and connect, say, the $i$ th recon-switches of adjacent processors in this ordering via their $h$-inputs and -outputs to form a chain ${ }^{1}$; the spare processor is at the end of the chain.

Fig. 4a shows such a recon-switch interconnection for a $4 \times 4$ array of processors (this array can correspond to a multiprocessor of any topology-most homogeneous or almosthomogeneous multiprocessors like $k$-ary $n$-cubes can be optimally laid out so that processors appear in regularly spaced rows and columns) in which each row and column corresponds to a distinct processor group. Thus, each processor belongs to exactly two processor groups, and this grouping is called a degree-two grouping. Fig. 8a shows a similar processor grouping and recon-switch interconnection for an FT four-dimensional hypercube. Note also that

1. It may not always be possible to connect all processors in a group by their $i$ th recon-switches throughout, in which case, after connecting the first few processors by their $i$ th recon-switches, we shift to connecting the rest via, say, their $j$ th recon-switches; we also connect the last $i$ th recon-switch of the first subset of processors to the first $j$ th recon-switch of the second subset of processors. each $P_{i}$ can be made two-FT by just attaching a second spare at the head end of this chain (the first spare processor is considered to be at the tail end of the chain), without requiring any extra recon-switches; see the second row of Fig. 4c, which has two spares $s_{*, 1}^{h}$ and $s_{*, 1}^{t}$ at either end-the labeling conventions for spares and groups will be specified in Section 5. When there are two faults in $P_{i}$, each can be reconfigured by covering sequences that go in opposite directions in $P_{i}$, each toward the nearer spare processor.

The underlying CG of Fig. 4a is shown in Fig. 4b. There are two salient points to note about such a CG for intersecting processor groups:

1) The covering arcs in the CG are bidirectional, i.e., the covering relation is symmetric (if $u \succ v$, then $v \succ u$ ). This is because the recon-switches are interconnected using bidirectional recon-links so that, in Fig. 4a, processor $(2,1)$ can replace processor $(1,1)$ or vice versa. Also, note in Fig. $4 \mathrm{c}$ that the covering sequences or reconfiguration paths for faults $(1,0)$ and $(2,1)$ are in opposite directions.

2) The covering relation within each processor group is also transitive, i.e., if $u \succ v$ and $v \succ w$, where $u, v$, and $w$ belong to the same processor group, then $u \succ w$. Again, this is facilitated by the switching structure. For example, in Fig. $4 c$, processor $(0,1)$ directly replaces processor $(2,1)$ (since $(1,1)$ is faulty) by passing through processor $(1,1)$ 's zeroth recon-switch. Note that if $(1,1)$ was nonfaulty, then bypassing it in this manner does not hinder its replacing capabilities in its column group, i.e., it can replace any processor in column $\# 1$ or can be replaced by any processor in it. This is because $(1,1)$ is bypassed by using only the $h$-input and $h$-output links of its recon-switch corresponding to its row group, and these links are not useful for $(1,1)$ to replace or be replaced by processors in its column group; see Fig. 4c. It is in this sense that the covering relation within a group is transitive, i.e., if $u$ replaces a nonadjacent processor in group $P_{i}$, then this does not inhibit the replacement capabilities of the bypassed intermediate processors in their other groups.

We thus have the following result.

THEOREM 1. The covering relation in a CG for any (disjoint or nondisjoint) processor grouping scheme in which each group is made $k_{1}-F T$, for any $k_{1} \geq 1$, is symmetric and, within each group, it is transitive. 


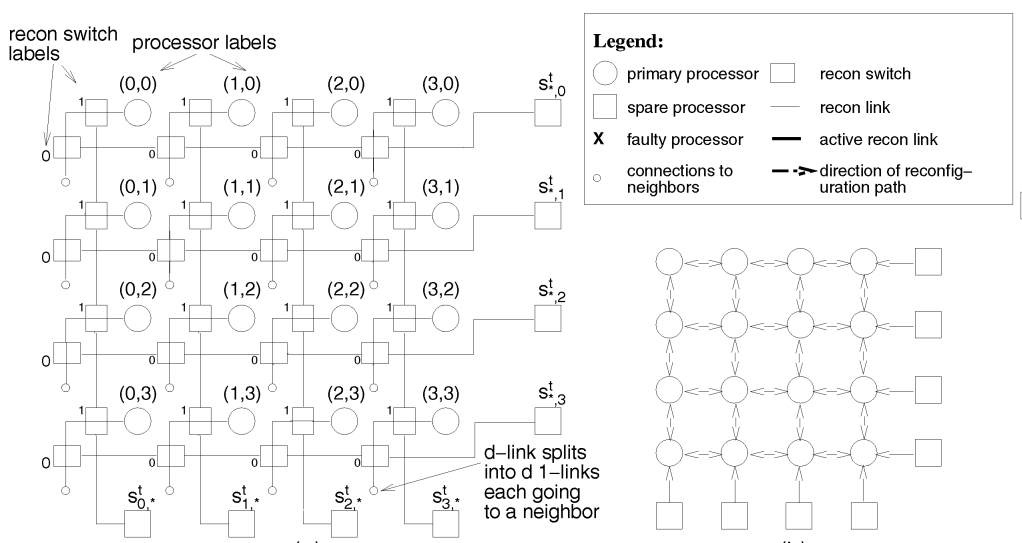

(a) (b)

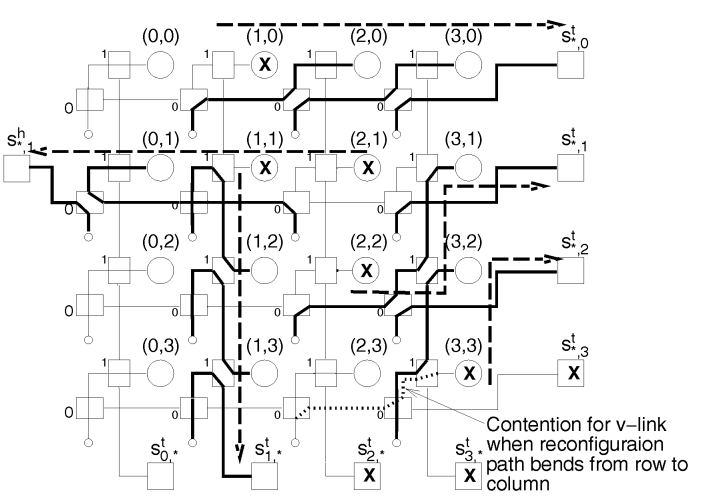

(c)

Fig. 4. (a) Recon-switch interconnections for intersecting groups along rows and columns; this processor grouping is also the one derived from a 2D-parity ECC. (b) Its covering graph. (c) Illustration of various reconfiguration paths.

\subsection{Reconfiguration}

We next characterize the fault patterns that can be reconfigured around in a design with intersecting groups. We first define the concept of matching. A matching system is set up as follows. There are two sets: $\mathcal{A}$ of size $n$ and $\mathcal{B}$ of size m. With each element $a_{i}$ of $\mathcal{A}$, a subset $B_{a_{i}}$ of $\mathcal{B}$ is associated. A matching is said to exist between $\mathcal{A}$ and $\mathcal{B}$ with respect to the collection of subsets $\left\{B_{a_{i}}: a_{i} \in \mathcal{A}\right\}$ if there is a one-to-one function $\phi: \mathcal{A} \rightarrow \mathcal{B}$ such that $\phi\left(a_{i}\right) \in B_{a_{i}}$, for all $i=1, \ldots, n$. The following theorem, known as Hall's theorem, states the condition under which a matching exists [3].

THEOREM 2 [3]. There exists a matching between sets $\mathcal{A}=\left\{a_{1}, \ldots\right.$, $\left.a_{n}\right\}$ and $\mathcal{B}=\left\{b_{1}, \ldots, b_{m}\right\}$ if and only if, for any $k$ elements $\left\{a_{i_{1}}, \ldots, a_{i_{k}}\right\}$ of $\mathcal{A}, \bigcup_{j=1}^{k} B_{a_{i_{j}}}$ has at least $k$ elements (of $\mathcal{B}$ ), for all $k \in\{1,2, \ldots, n\}$.

The next theorem establishes that a matching between the set of faults and the set of spares in the above sense is sufficient for reconfiguration.

THEOREM 3. Let $F$ be the set of processor faults. For each primary fault $u$, let $S_{u}$ be the set of nonfaulty spares in each group that $u$ belongs to, and, for each spare fault $v$, let $S_{v}=\{v\}$. Then, if there is a matching between $F$ and the set $S$ of all spares, with respect to $\left\{S_{u}: u \in F\right\}$, then the set of faults $F$ is reconfigurable.

PROOF. Let $s_{u}$ be the spare matched to primary fault $u$ by the mapping $\phi: F \rightarrow S$ (note that we are not interested in reconfiguring a faulty spare which is mapped to itself by $\phi)$. Then, since, from Theorem 1 , the covering relation within each group is transitive, and since each spare is associated with a distinct group, (in the worst-case) $s_{u}$ can directly replace $u$ without disturbing any intermediate processor's replacement capabilities in other groups. ${ }^{2}$

2. Practically speaking, having the spare directly replace a distant processor in its group is not such a good idea, since this makes the links from $s_{u}$ to its new neighbors (the previous neighbors of $u$ ) very long. Thus, each fault should be directly replaced by the nearest available nonfaulty processor in
While matching is sufficient for reconfiguration, it is not necessary. For example, in Fig. 4c, there is no matching for the set of primary faults $\{(1,0),(1,1),(2,1),(2,2),(3,3)\}$, since both spares in $(3,3)^{\prime} \mathrm{s}$ two groups are faulty. However, reconfiguration is still possible as shown in the figure. In this case, $(3,3)$ is replaced by $(3,2)$, which, in turn, is replaced by spare $s_{*, 2}^{t}$ resulting in a "bend" in the reconfiguration path. This bend occurs because $(3,2)$ 's replacement of $(3,3)$ is along its column group and then $s_{*, 2}^{t}$ 's replacement of $(3,2)$ is along its row group. We are thus bending from a group connected by a higher numbered reconswitch (\#1) to one connected via a lower numbered reconswitch (\#0). The reverse type of bend, i.e., replacement along a row followed by replacement along a column is not possible because of the contention for the $v$-link connecting the first and zeroth recon-switch of the processor at which the bend occurs. Finally, note in the figure that $(1,0),(1,1)$, and $(2,1)$ are all replaced by "matched" spares in their own groups, and their reconfiguration paths are thus straight.

It is possible to exactly determine a valid set of reconfiguration paths for a set of faults in the above-described switching structure using a network max-flow algorithm, as described later in Section 6. However, the matching criterion is still important because

1) In most cases, we can determine the reconfiguration paths by using a simple matching algorithm,

2) It points us to the use of ECCs as a systematic method for determining FT processor groups, and

3) It is also useful in obtaining the deterministic fault tolerance of our designs.

\section{Using ECCs to Determine FT Processor GROUPS}

\subsection{Introduction}

A linear ECC has $N$ information bits and $g$ check-bits that are related to the information bits by linear or parity equations,

the group (to which $s_{u}$ belongs), which should also be similarly replaced, and so on, until $s_{u}$ replaces a processor in this covering sequence for $u$. Only in the worst case, for example, when all intermediate processors are faulty, will $s_{u}$ need to directly replace $u$. 


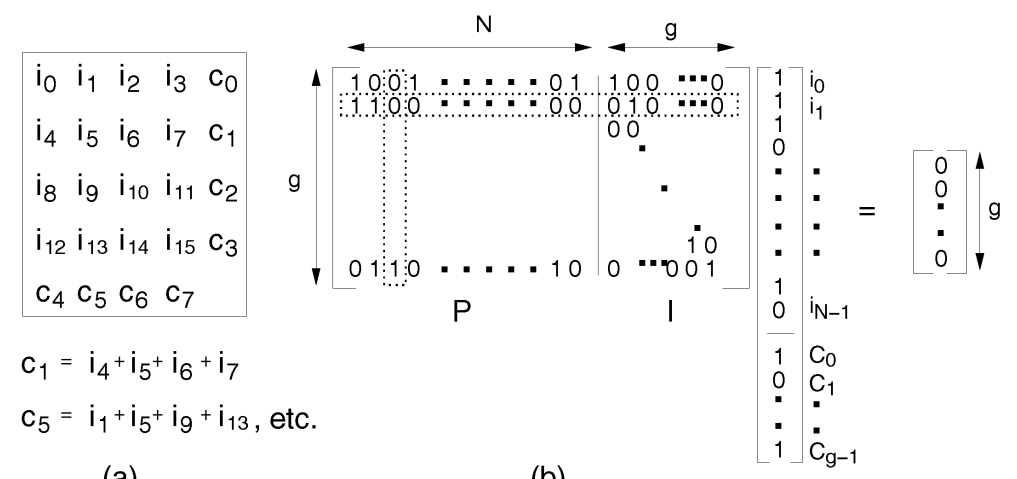

(a)

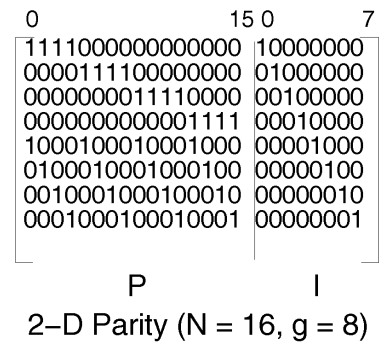

(c)

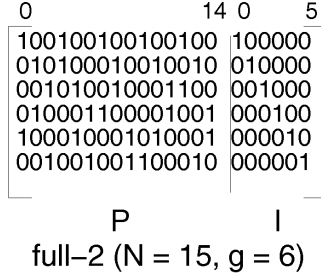

(d)

Fig. 5. (a) Information bit groups in the 2D-parity code. (b) A parity check matrix $H=[P \mid /$ for a linear code with $N$ information and $g$ check-bits. Multiplying $H$ with a valid code word $X$ results in a vector of zeros.

and can be used to determine if there are any errors in the code word (the combination of information and check-bits), and possibly correct them. The simplest linear ECC (hereafter referred to only as ECC) is the single- or 1Dparity code in which the single check or parity bit

$$
c_{0}=\sum_{t=0}^{N-1} i_{t}
$$

where the $i_{t} s$ are the information bits and $\sum$ and + denote the modulo-2 sum or Exclusive-Or (XOR) operation. In the rest of this section, all arithmetic will be modulo 2; note that modulo-2 multiplication is the same as the AND operation. A single error in any bit in the code word will result in a violation of the above equation and thus lead to a detection of the error. Instead of talking about the parity of all the information bits, we can talk about parities of various groups of information bits. An example is the 2D-parity code, in which the $N$ information bits are arranged in two dimensions as an $n \times m$ array, where $N=n \cdot m$, and each row and column of this matrix is a group with which a parity check-bit is associated; see Fig. 5a, in which two example parity equations (for $c_{1}$ and $c_{5}$ ) are also given. The 2D-parity code can detect any two errors (its deterministic error detectability) and can correct a single error.

Any (linear) ECC is essentially a collection of parity equations, one for each group of information bits. We can construct processor groups from a given ECC in the following manner. Each primary processor is uniquely associated with an information bit and each spare processor with a check-bit. Each group and its check-bit in the ECC then gives rise to a processor group and its associated spare. ${ }^{3}$

There are five metrics associated with ECCs that are of interest to FT multiprocessor design:

1) Check-bit overhead, which is the ratio of check to information bits. This translates to spare-processor overhead in an FT multiprocessor. If the 2D arrangement is an $n \times n$ square, i.e., $N=n^{2}$, then this overhead

3. Note that the two-spare- per-group assignment discussed in Section 3 does not exactly fit an ECC structure (since there is no point in having two check-bits per information-bit group, both with the same parity equations). However, it is a natural augmentation of the one spare per processor group structure derived from the ECC that leads to higher reliability and higher utilization of the recon-switches and links in the FT multiprocessor. is $g / N=2 n / N=2 / \sqrt{N}$.

2) Group size, which is the size of the largest group. This is important, since, when a single fault occurs that can be reconfigured using matching (to a spare in one of its groups), then only the processors in that group need be involved in the replacement process, and will not be available for the period it takes to reconfigure. Thus, with a large group size, the availability of the multiprocessor decreases. Group size of the square 2D-code is $\sqrt{N}$.

3) Group degree, which is the maximum number of groups an information bit lies in. In an FT multiprocessor, this translates to the maximum number of recon-switches and recon-links needed per processor. The 2D-parity code has degree two.

4) Deterministic error detectability (DED), which is the maximum number of errors that the ECC will always detect. This provides a lower bound on the DFT (maximum number of processor faults that can always be reconfigured around) of the FT multiprocessor, as we will shortly see.

5) Average erasure correctability, which is defined similarly to average fault tolerance $k_{\text {avg }}$ (see Section 1). The concept of erasure correctability, which was introduced in [18], will be explained shortly. This metric provides a lower bound on the average fault tolerance of the multiprocessor, as shown later.

We will describe four useful ECCs, the 2D-parity, fulltwo, 3D-parity, and full-three codes, that provide different trade-offs of these metrics. The partitioned 1D-parity code yields the disjoint-group or local sparing FT design discussed briefly in Section 3; however, as we will see in Section 8 , its features are not as attractive as designs based on other ECCs that result in intersecting FT processor groups. Before describing these other codes, we briefly revisit the theory of error-correcting codes [1] that will be useful in formulating why ECCs are a good basis for determining FT processor groupings.

\subsection{Relating Error Detection, Erasure Correction, and Fault Tolerance}

A linear ECC can also be described in terms of a $g \times(N+g)$ parity check matrix, $H=[P \mid I]$ shown in Fig. $5 \mathrm{~b}$. The $N$ columns of $P$ represent information bits, and the $g$ columns of 
I the check-bits. Each row corresponds to a group; the columns that have $1 \mathrm{~s}$ in a row correspond to information bits in that group. If vector $X$ is a valid codeword, i.e., its checkbits are derived from the information bits using the parity equations, then $H \cdot X=0$ (recall that we are using modulo-2 arithmetic). We next define the concept of linear independence and then state a basic theorem in error-correction coding theory. A set $S$ of binary vectors is said to be linearly dependent, if the modulo-2 sum of some subset of its vectors yields the 0 vector. If $S$ is not linearly dependent, then it is said to be linearly independent.

THEOREM 4 [1]. The DED of an ECC is $t$ if and only if any $t$ columns of its parity matrix are linearly independent.

Similar to the matching system between faulty processors and spares described in Section 3, we can set up a matching system between bits and parity groups as follows. Let $A$ be the set of information and check-bits $i_{0}, i_{1}, \ldots, i_{N+g}$ and $\mathcal{P}$ the set of parity groups in a code. With each bit $i_{j}$ in $A$, we associate a subset $P_{i_{j}}$ of $\mathcal{P}$ of those parity groups that contain $i_{j}$. Then, given a subset $E$ of erroneous bits of $A$, there is a matching between $E$ and $\mathcal{P}$, with respect to $\left\{P_{i_{j}}\right.$

$\left.: i_{j} \in E\right\}$, if there exists a one-to-one function $\psi: E \rightarrow \mathcal{P}$, such that $\psi\left(i_{j}\right) \in P_{i_{j}}$ for all $i_{j} \in E$. We next relate matching and error detectability of a code; we first state a useful result from linear algebra [17] given here in a more relevant form.

THEOREM 5 [17]. Any set of $m$ vectors, each of length $n$, are linearly dependent if $m>n$.

THEOREM 6. If $t$ is the DED of a code $C$, then there will always be a matching between any set $E$ of at most $t$ erroneous bits and the set $\mathcal{P}$ of parity groups of $C$.

Proof. Suppose there is a subset $E$ of $t$ bits for which there is no matching. Since $C$ can detect any $t$ errors, it means that the columns of the parity matrix $H$ corresponding to the bits in $E$ are linearly independent (Theorem 4). For convenience, let these columns be $H^{0}, \ldots, H^{t-1}$. Note that the set $P_{i_{j}}$ is the set of rows (i.e., parity groups) in which the $i_{j}$ th column of $H$ has 1 s. Since there is no matching for bits $i_{0}, \ldots i_{t-1}$, from Theorem 2, there is a nonempty subset $E^{\prime}$ of $E$ of size $m \leq t$ whose bits are all contained within $l \leq m-1$ parity groups. In other words, the $m$ columns in $H$, say, $H^{0}, \ldots, H^{m-1}$, corresponding to the bits in $E^{\prime}$, together have $1 \mathrm{~s}$ in $l \leq m-1$ rows and $0 \mathrm{~s}$ in all other rows. To determine if these columns are linearly dependent, we need only consider these $l$ rows, since their sum will yield $0 s$ in the other $g-l$ rows. Thus, we can only consider the $m$ subcolumns of $H^{0}, \ldots, H^{m-1}$ of length $l$ that contain only the rows in which at least one of them has a 1 . Since $l<m$, it follows from Theorem 5 that these subcolumns are linearly dependent, and, thus, $H^{0}, \ldots, H^{m-1}$ are linearly dependent. Hence, $H^{0}, \ldots, H^{t-1}$ are also linearly dependent and we reach a contradiction.

THEOREM 7. The DFT of a multiprocessor whose FT processor groups are based on ECC C is lower bounded by C'S DED when there is one spare per processor group, and by twice its $D E D$ when there are two spares per group.

Proof. Let $C$ be $t$ error detecting. Then, from Theorem 6, there is a matching for any $t$ errors. We first consider the single spare-per-group design. Since each bit corresponds to a primary or spare processor in the multiprocessor, and since the bit-to-parity equation matching system corresponds to that between faulty processors and the set of spares, there exists a matching in the latter system for any $t$ faults. Hence, from Theorem 3 , the $t$ faults can be reconfigured around. Now, consider the two-spare-per-group design, and suppose the multiprocessor has $2 t$ faults; we randomly partition them into two size- $t$ sets $F_{1}$ and $F_{2}$. From the above discussion, the faults in $F_{1}$ can be matched to the tail spares in the system (each group has a spare at the tail of the group, as in the one-spare case, and a second spare at its head). Note that the presence of fault set $F_{2}$ does not hinder the reconfiguration of the faults in $F_{1}$, since, if the former are in the matching-based reconfiguration paths of the latter, then they are simply bypassed. Similarly, fault set $F_{2}$ can be matched to the head processors in the system. In this case, however, the reconfiguration path from any $u \in F_{2}$ to its matched head spare in, say, group $i$, might conflict or overlap with that of a fault $v \in F_{1}$ that is matched to the tail spare in group $i$. This happens when $u$ is nearer to the tail spare and $v$ nearer to the head spare in group $i$. Thus, the matched spares of $u$ and $v$ can be interchanged, and $u$ can be reconfigured to the tail spare and $v$ to the head spare in group $i$ without conflict. Note that there are at most two reconfiguration paths in a group, the above interchange does not affect any reconfiguration paths in any other group, and this type of interchange can be made in every group in which there are conflicts between two reconfiguration paths.

In Section 7, we analyze the exact DFT of multiprocessors based on various codes and show that these are quite higher than the respective DEDs of these codes.

In general, if $r>t$ errors occur, then they are detectable as long as the sum of the $r$ columns corresponding to $r$ error bits is nonzero [1]. Linear independence of the $r$ columns is sufficient to yield a nonzero sum; however, it is not necessary (for example, a subset of the $r$ columns can be summed to yield a 0 vector, but the sum of all $r$ columns may not be zero). A more relevant concept for our purpose is erasure correctability of a code used in [18], where ECCs were applied to design redundant disk arrays (RAIDs) in which disk erasures (disk failures in which the data on the disk is lost) can be tolerated by reproducing the data of the failed disks using parity equations. In such RAIDs, each primary (spare) disk corresponds to an information (check) bit of the 
code. An erasure is essentially a loss of bits in a code word whose locations are known but not values. If these unknown or lost bits can be reproduced, then we say that the erasure is correctable. The deterministic erasure correctability of a code is the same as its DED [18]. However, its average erasure correctability is upper-bounded by its average error detectability, since $r>t$ disk erasures can be corrected if and only if the $r$ columns corresponding to the failed disks are linearly independent [18]. The mean-time-tofailure (MTTF) of a RAID derived from an ECC was determined in [18], and this gives a reasonably good measure of the average erasure correctability of the code. The next theorem relates the average fault tolerance of an FT multiprocessor to the average erasure correctability of a code.

THEOREM 8. The average number of erasures that can be corrected by a code $C$ is a lower bound for the average number of faults that can be tolerated by an FT multiprocessor whose processor groupings, with one spare per group, are based on $C$.

PROOF. Suppose there are $r>t$ errors (erasures in the corresponding RAID) in a code word. These erasures can be corrected if the corresponding $r$ columns of the parity matrix $H$ are linearly independent. Proceeding in the same manner as in the proof of Theorem 7, we can see that, in such a case, the corresponding $r$ processor faults in the FT multiprocessor can be reconfigured to matched spares. Thus, for each correctable pattern of disk erasures, there is a corresponding pattern of reconfigurable processor faults. The theorem follows from this observation.

We obtain the following useful corollary of this theorem.

COROLLARY 1. The reliability (MTTF) of a code C or of the corresponding RAID system, as determined by its erasure correctability, is a lower bound for the reliability (MTTF) of the corresponding FT multiprocessor, with one spare per group, if we assume that the failure probability of a disk in the RAID system is the same as that of a processor. Also, the above metrics for a two-spare-per-group design are lower bounded by the respective metrics of the corresponding single-spare-per-group design.

In [18], a number of useful ECCs like the 2D-parity, 3Dparity, full-two, and full-three codes were identified that yield very high MTTF for RAID designs based on these codes, and which have attractive values of metrics, like check-bit overhead, group degree, and group size. These metrics are also relevant to FT multiprocessor design as discussed earlier. In view of this, and Theorems 7 and 8 and Corollary 1, we choose to use these codes to design FT multiprocessors. Later, we show that the DFT, layout-area overhead, and reliability of these FT multiprocessors are extremely favorable. Next, we briefly describe the above ECCs.

\subsection{Some Useful ECCs}

The 2D-parity code was described earlier; its parity matrix is shown in Fig. 5c. The 3D-parity code can be similarly formed by arranging the information bits in a threedimensional array and associating a parity equation with each dimensional group. Its check-bit overhead is $3 / N^{1 / 3}$, its group size is $N^{1 / 3}$, it is a degree-three code and its DED is three. The full-two code is a degree-two two-DED code. It can be defined in terms of its parity matrix $H_{\text {full } 2}=\left[P_{\text {full } 2} \mid I\right]$. $P_{\text {full } 2}$ consists of all possible distinct columns with exactly two $1 \mathrm{~s}$; see Fig. $5 \mathrm{~d}$. The number $N$ of information bits in terms of $g$ is given by $N=g(g-1) / 2$, i.e., its check-bit overhead is approximately $\sqrt{2 / N}$, and its group size is $g-1 \approx \sqrt{2 N}-1$. The full-three code is a degree-three three-DED code, and $P_{\text {full } 3}$ consists of all possible distinct columns with exactly three 1s. For this code, $N=g(g-1)(g-2) / 6$, thus, its checkbit overhead is approximately $(6 N)^{1 / 3} / N=1.8 / N^{2 / 3}$, and its group size is $3 N / g=(g-1)(g-2) / 2 \approx(6 N)^{2 / 3} / 2$. The fulltwo and full-three codes have the least possible check-bit overheads of any degree-two and degree-three codes, respectively. The 2D- and 3D-parity codes have better reliabilities than the full-two and full-three codes, respectively, though, obviously, higher check-bit overheads. However, as we will see next, wiring overheads for FT multiprocessors based on full-two and full-three codes are a little higher than those based on the 2D- and 3D-parity codes, respectively.

\section{WiRe-EfFicient Layouts}

\subsection{Layout Scheme}

In this section, given a regular 2D-square or 3D-cubic layout $^{4}$ of a non-FT multiprocessor of an arbitrary topology, we will obtain a similar layout of an ECC-based FT multiprocessor by embedding processor groupings defined by the ECC in the given non-FT processor grid. Embeddings with minimum edge congestion (the number of recon $d$-links that get mapped onto a single grid line, i.e., the space between adjacent processor rows/columns/heights) will be used to minimize the redundant wiring overhead, and thus obtain efficient FT layouts in a topology independent manner. Let $0,1, \ldots, g-1$ be the $g$ processor groups in the FT multiprocessor and let $d_{g}$ denote its group degree (the number of groups a processor belongs to). Any processor $u$ is associated with a $d_{g}$-digit label $\left(b_{0}, b_{1}, \ldots, b_{d_{g}-2}, b_{d_{g}-1}\right)$ which specifies its location in a $d_{g}$-dimensional grid-for different codes, these labels will be determined differently. Recall from Section 3 that every processor uses $d_{g}$ recon-switches connected in series and labeled $0,1, \ldots, d_{g}-1$. (see, e.g., Fig. 4a).

For the 2D- and 3D-parity codes, the processor labels are the subsets of grid points given by the square $\left\{\left(b_{0}, b_{1}\right): 0 \leq b_{0}, b_{1}<\sqrt{N}\right\}$ and the cube $\left\{\left(b_{0}, b_{1}, b_{2}\right): 0 \leq b_{0}, b_{1}\right.$, $\left.b_{2}<N^{1 / 3}\right\}$, respectively. Processors in the same row have different $b_{0}$ but the same $b_{1}$ and $b_{2}$ (for the 3D-parity code) values, and belong to the same group. These processors are linked from the smallest- (the head processor) to the largest-labeled processor (the tail processor) via their zeroth

4. Actually, as will become clear later, our layout schemes are equally effective when the given non-FT layout is rectangular or cuboidal. 

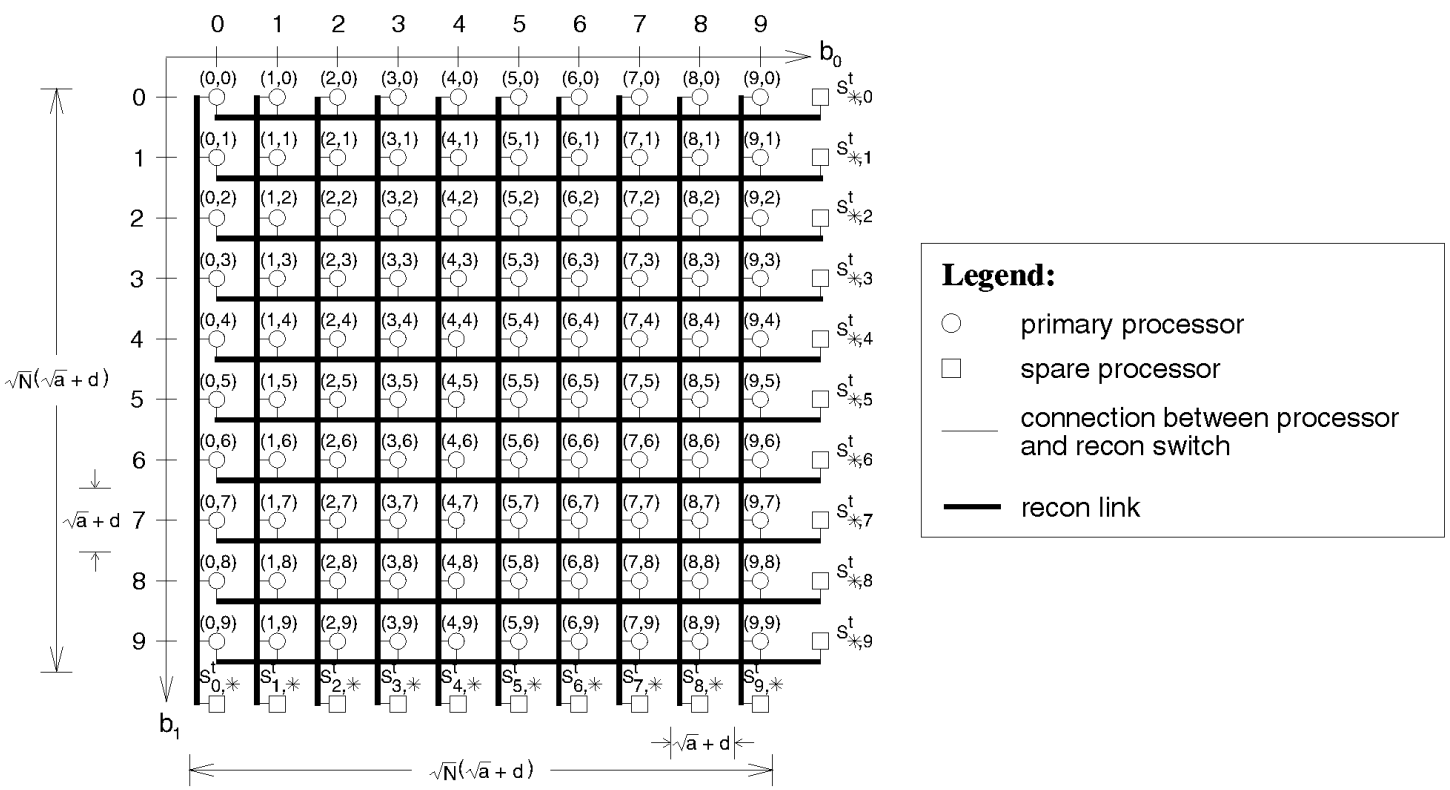

Fig. 6. Wire-efficient embeddings of processor groupings in an FT multiprocessor array (of any topology) derived from the 2D-parity code for $N=$ 100 and $g=20$.

recon-switch. In a single-spare-per-group design, we connect a spare $s_{*, b_{1}, b_{2}}^{t}$ to the tail processor in the group, and, in the double-spare case, we connect, in addition, another spare $s_{*, b_{1}, b_{2}}^{h}$ to the head processor via their zeroth reconswitches. Similarly, column and height groups are formed, and have spares connected to them. Note that this layout has an edge congestion of one (see Fig. 6).

Next, for the full-two and full-three codes, the label $\left(b_{0}, b_{1}, \ldots, b_{d_{g}-2}, b_{d_{g}-1}\right)$ of a processor $u$ indicates, in addition to its position, the groups to which $u$ belongs, so that $0 \leq b_{i}$ $<g$ for each $b_{i}$. Moreover, now the $i$ th recon-switch is used to connect $u$ to group $b_{i}$. Note that any two or three groups in the full-two and full-three codes, respectively, contain exactly one common processor. Suitable subsets of grid points for these two codes that satisfy this requirement and that give compact 2D and 3D triangular pyramidal layouts of processors, respectively, are $\left\{\left(b_{0}, b_{1}\right): g>b_{0}>b_{1} \geq 0\right\}$ (see Fig. 7a) and $\left\{\left(b_{0}, b_{1}, b_{2}\right): g>b_{0}>b_{1}>b_{2} \geq 0\right\}$-these layouts will be modified to square and cubic layouts, respectively, in Section 5.2.

We interconnect processors to form groups as follows. For any group $j$, where $0 \leq j<g$, let $j_{\max }$ and $j_{\min }$ be the largest and smallest digit positions, respectively, over all processors in the group, at which the group number $j$ appears in a processor's label. For instance, in Fig. 7a, for group $j=0$, $j_{\max }=j_{\min }=1$, and, for group $j=8, j_{\max }=1$ and $j_{\min }=0$. The $j$ th processor group is formed by first linking all processors with $b_{k}=j$, where $j_{\min } \leq k \leq j_{\max }$, starting from the first to the last processor in a $\left(d_{g}-1\right)$-dimensional radix-g Gray-code ordering (obtained as an extension of reflected binary Gray codes) of their corresponding $\left(d_{g}-1\right)$-digit labels $\left(b_{0}, \ldots\right.$, $b_{k-1}, *, b_{k+1}, \ldots, b_{d_{g}-1}$, via their $k$ th recon-switches. For instance, if $g=8, d_{g}=3, k=1, j=3$, and the set of processors with $b_{1}=3$ is $\{(4,3,0),(4,3,1),(4,3,2),(5,3,0),(5,3,1),(5,3,2)$, $(6,3,0),(6,3,1),(6,3,2),(7,3,0),(7,3,1),(7,3,2)\}$, then the two-dimensional radix-8 Gray-code ordering of the corresponding labels $\left(b_{2}, b_{0}\right)$ is $(4,0),(4,1),(4,2),(5,2),(5,1)$, $(5,0),(6,0),(6,1),(6,2),(7,2),(7,1),(7,0)$.

In the case of the full-three code, we actually order the processors in an almost-Gray-code manner, since not all $\left(d_{g}-1\right)$-digit labels above appear in processor labels. Consider group 0 (i.e., $j=0$ ) and $k=2$. First, we order processors with labels of the form $(2, *, 0)$ from the smallest to the largest labeled processor-processor $(2,1,0)$ is the only such processor. Then, we order all processors with labels of the form $(3, *, 0)$ from the largest to the smallest labeled processor and add them to the previous ordering of processors-processors $(3,2,0)$ and $(3,1,0)$ are the only such processors and are arranged in that order. Next, processors with labels of the form $(4, *, 0)$ are ordered from the smallest to the largest labeled and appended to the above ordering-thus, the sequence $(4,1,0),(4,2,0),(4,3,0)$ is appended to the above ordering, and so forth. A Gray-code (or almost-Gray-code) ordering, like the ones above, is useful in minimizing reconfiguration link length between adjacent processors in a group-recall that a processor's label gives its location in a $d_{g}$-dimensional grid, and, thus, adjacent processors in this ordering will be spatially adjacent.

Next, the last processor (in the above ordering) with $b_{k}=j$ is linked to the first processor with $b_{k+1}=j$, for $j_{\min } \leq k<j_{\max }$, via the $k$ th recon-switch of the former and the $(k+1)$ th recon-switch of the latter. This gives us a total ordering of all the processors in group $j$ with the first processor with $b_{j_{\min }}=j$ at the head of the group and the last processor with $b_{j_{\max }}=j$ at the tail of the group. For instance, in the 


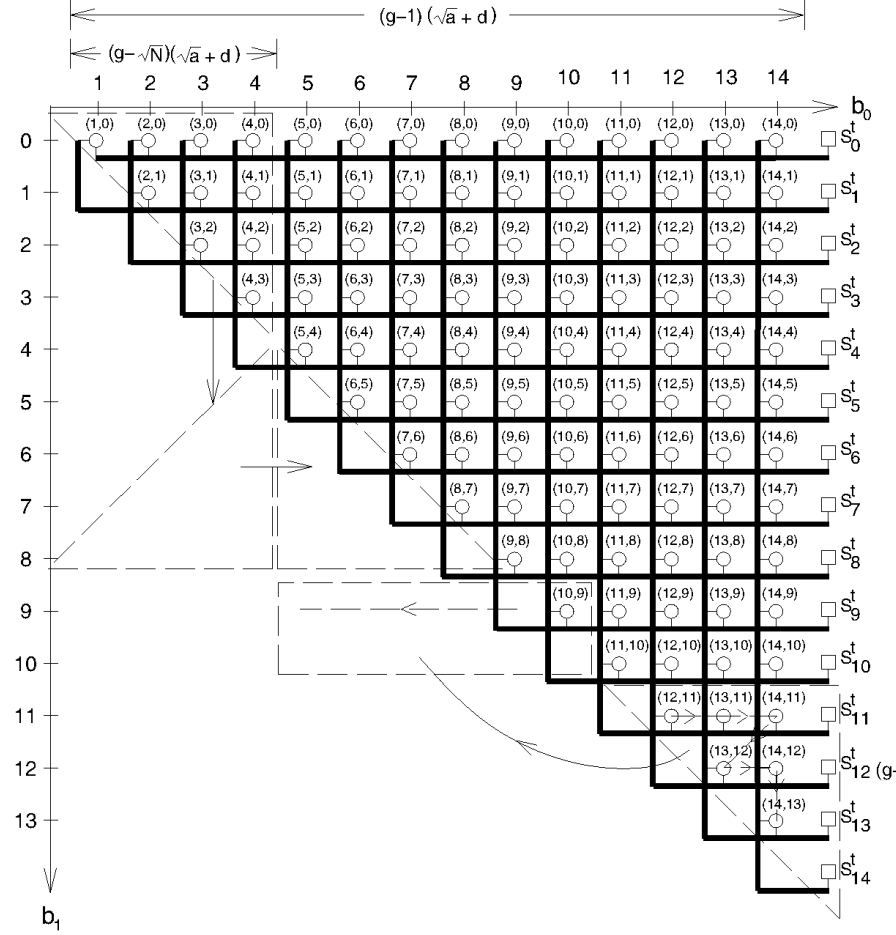

(a)

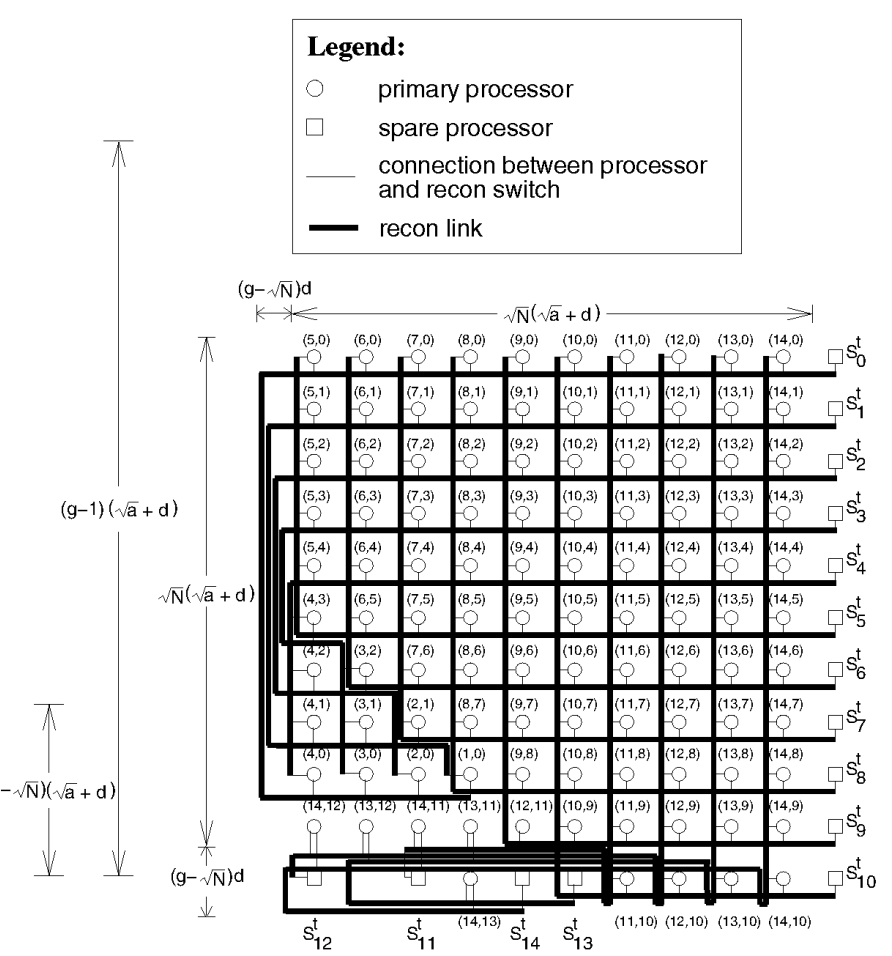

(b)

Fig. 7. Wire-efficient embeddings of processor groupings in an FT multiprocessor array (of any topology) derived from the full-2 code in a (a) triangular 2D layout and (b) in a square 2D layout obtained by modifying the triangular layout, for $N=105$ and $g=15$.

full-two code based FT multiprocessor in Fig. 7a, group 8 is formed by first linking processors $(8,0),(8,1), \ldots,(8,7)$ in that order via their zeroth recon-switches and processors $(9,8),(10,8), \ldots,(14,8)$ in that order via their first reconswitches, and then linking processor $(8,7)$ to $(9,8)$ via the former's zeroth and the latter's first recon-switches. The head and tail processors in this group are processors $(8,0)$ and $(14,8)$, respectively. We connect spares $s_{j}^{t}$ and $s_{j}^{h}$ to the tail and head processors, as in the 2D- and 3D-parity cases, via the $j_{\max }$ th and $j_{\min }$ th recon-switches, respectively. Note, again, that the layouts obtained have a congestion of one.

\subsection{Area/Volume Overhead}

We now give the fractional area (volume) overhead $f_{o}$ of the 2D (3D) layouts of FT designs based on the various codes. Denote the area (volume) of the non-FT multiprocessor by $A$ and let $a=A / N$ be the area (volume) per processor-this area (volume) is assumed square (cubic) and includes the area (volume) occupied by the actual processor and its share of the interprocessor wiring. Recall that the recon $d$ link for each group corresponds actually to $d$ sets of wires, one for each neighbor of a processor, so that its width is $d$ units.

THEOREM 9. The fractional area overhead of an FT design based on the 2D-parity code compared to a non-FT design is $f_{0}=\frac{2 \sqrt{a} d+d^{2}}{a}$.

PROOF. The area of the FT design is

$$
(\sqrt{N} \cdot(\sqrt{a}+d))^{2}=N \cdot\left(a+2 \sqrt{a} d+d^{2}\right)
$$

(see Fig. 6). Hence, $f_{o}=\frac{N \cdot\left(a+2 \sqrt{a} d+d^{2}\right)-N a}{N a}=\frac{2 \sqrt{a} d+d^{2}}{a}$.

THEOREM 10. The fractional area overhead of an FT design based on the full-two code compared to a non-FT design is $f_{o} \approx \frac{2 \sqrt{a} d+d^{2}}{a}$ for a triangular layout and $f_{o} \approx \frac{2 \sqrt{a} d+2 d^{2}}{a}$ for a square layout.

PROOF. Recall that for a full-two code $\frac{(g-1)^{2}}{2} \approx \frac{g(g-1)}{2}=N$, so that $g \approx(g-1) \approx \sqrt{2 N}$. First, consider a triangular layout, as in Fig. $7 \mathrm{a}$, that has a base and height of $g-1$ processors each. The area of the FT design is

$$
\frac{1}{2} \cdot((g-1)(\sqrt{a}+d))^{2} \approx N \cdot\left(a+d^{2}+2 \sqrt{a} d\right),
$$

thus giving $f_{o} \approx \frac{2 \sqrt{a} d+d^{2}}{a}$.

A square layout of the FT design can be obtained by modifying the triangular layout of Fig. $7 \mathrm{a}$ as shown in Fig. $7 \mathrm{~b}$. The polygon of processors $((5,0)$, $(14,0),(14,10),(11,10),(5,4))$ and the associated spares on the right in Fig. 7a remain fixed. The triangle of processors $((1,0),(4,0),(4,3))$ is inverted, flipped, and placed at the bottom left corner of the above polygon. The triangle of processors $((12,11)$, $(14,11),(14,13))$ is scanned in row-major order and placed right to left at the bottom of the above layout, as shown in Fig. $7 \mathrm{~b}$. Spare processors $s_{11}^{t}, s_{12}^{t}, s_{13}^{t}$, and $s_{14}^{t}$ are placed appropriately in the last row. For any $N$, the above scheme can be used to obtain a square layout of $\sqrt{N}$ processors to a side, with an edge congestion of $(g-\sqrt{N})+1$ in the leftmost column and 
bottommost row (corresponding to recon links connected to $(g-\sqrt{N})$ groups in the two displaced triangle of processors above) and one in all other rows and columns (see Fig. 7b). Therefore, the area of the square FT layout is

$$
\begin{aligned}
(\sqrt{N}(\sqrt{a}+d)+(g-\sqrt{N}) d)^{2} & \approx(\sqrt{N} \sqrt{a}+\sqrt{2 N} d)^{2} \\
& =N\left(a+2 \sqrt{2 a} d+2 d^{2}\right),
\end{aligned}
$$

so that $f_{0} \approx \frac{2 \sqrt{2 a} d+2 d^{2}}{a}$.

THEOREM 11. The fractional volume overhead of an FT design based on the 3D-parity code compared to a non-FT design is $f_{0}=\frac{3 a^{2 / 3} d+3 a^{1 / 3} d^{2}+d^{3}}{a}$.

PROOF. As in the 2D-parity code based design, with each processor group along a row, column, or height, we have a wiring overhead of $d$, so that the width of each row, column, or height is $\left(a^{1 / 3}+d\right)$. Thus, the volume of the FT design is $\left(N^{1 / 3} \cdot\left(a^{1 / 3}+d\right)\right)^{3}$, which implies $f_{0}=\frac{3 a^{2 / 3} d+3 a^{1 / 3} d^{2}+d^{3}}{a}$.

THEOREM 12. The fractional volume overhead of an FT design based on the full-three code, compared to a non-FT design, is $f_{o} \approx \frac{3 a^{2 / 3} d+3 a^{1 / 3} d^{2}+d^{3}}{a}$ for a triangular pyramidal layout and $f_{o} \approx \frac{3 \cdot 6^{1 / 3} a^{2 / 3} d+3 \cdot 6^{2 / 3} a^{1 / 3} d^{2}+6 d^{3}}{a}$ for a cubic layout.

PROOF. Since the redundant area overhead around each processor in the square 2D-parity code and triangular full-two code layouts is the same (see Figs. 6 and 7a), the corresponding overheads are also the same (see Theorems 9 and 10). The case is the same with the cubic 3D-parity code and triangular pyramidal fullthree code layouts. Thus, from Theorem 11, in the latter case, $f_{o} \approx \frac{3 a^{2 / 3} d+3 a^{1 / 3} d^{2}+d^{3}}{a}$.

The triangular pyramidal layout for the full-three code can be modified to obtain a cubic layout in a manner similar to that in the full-two code case considered in the proof of Theorem 10. Again, analogous to the full-two code case, the volume of a cubic full-three code layout is $\left(N^{1 / 3}\left(a^{1 / 3}+d\right)+\left(g-N^{1 / 3}\right) d\right)^{3}$. Since, for a full-three code, $\frac{g(g-1)(g-2)}{6}=N$, we approximate $g$ by $(6 N)^{1 / 3}$, obtaining $f_{o} \approx \frac{3 \cdot 6^{1 / 3} a^{2 / 3} d+3 \cdot 6^{2 / 3} a^{1 / 3} d^{2}+6 d^{3}}{a}$.

The total area or volume $A$ of a layout for a given network topology is proportional to the square or $3 / 2$ power, respectively, of its bisection width, which is defined as the minimum number of wires that must be cut to separate the network into two equal halves. The bisection widths for mesh and hypercube architectures are $\sqrt{N}$ and $N / 2$, so that their $a$ values are $\Theta(1)$ and $\Theta(N / 4)$, respectively. To get an idea of the area/volume overhead, let us consider an example. Recall that $d$ is the combined width of all redundant wires (i.e., a recon $d$-link) entering a processor when the wires are closely packed (as in the layouts of Figs. 6 and $7 \mathrm{~b})$, and $\sqrt{a}$ or $a^{1 / 3}$ is the width of a side of a square area or cubic volume, respectively, containing a processor, several memory chips, the processor-memory bus, possibly a separate routing chip and input/output lines, and the processor's share of nonredundant interprocessor wiring. Furthermore, note that $d / 4$ nonredundant wires enter each side of the square routing chip contained in this square region, and that a wire connected to a pin on the chip will have a much smaller width compared to the pin pitch. Therefore, a reasonable value for the ratio between the combined width of $d / 4$ sets of closely packed redundant wires and the side of an individual square region would be $\frac{d / 4}{\sqrt{a}}=0.05$ for a mesh, and $\frac{d / 4}{\sqrt{a}}=0.05 \frac{(\log N) / 4}{\sqrt{N / 4}}$ for a hypercube architecture, where note that $d=4$ for a mesh and $d=\log N$ for a hypercube. The wire width $d / 4$ is similarly related to the width $a^{1 / 3}$ of a cubic region for a 3D layout.

From the above theorems, we obtain the following results for a mesh architecture (of any size): $f_{o}$ (square 2Dparity or triangular full-two code layout) $=44$ percent and $f_{o}$ (square full-two code layout) $=65$ percent; for a 1,024processor hypercube the corresponding overheads are six percent and nine percent. The overheads for the 3D layouts corresponding to the 3D-parity and full-three codes are somewhat higher. For the mesh architecture, the overheads corresponding to the 2D-parity and full-two codes are reasonable considering the improvement in reliability of the system (as will be seen in Section 8), while, for the hypercube architecture, the corresponding overheads are quite low.

\section{Reconfiguration Algorithm}

The reconfiguration problem in the node-covering method is equivalent to finding the maximum flow in a flow graph G; such a correspondence was also observed in [29] for the FT design presented therein. The flow graph corresponding to the $4 \times 42$ D-parity code based FT array of Fig. $8 \mathrm{a}$ is shown in Fig. $8 b$ and, in the general case, is described as follows. For each recon-switch, there is a vertex in $G$, and, between recon-switches in the same or different processors that are linked, there are two oppositely directed edges to model reconfiguration in either direction between the corresponding switches. Furthermore, there is an edge from the topmost $\left(\left(d_{g}-1\right)\right.$ th) to the bottommost (zeroth) reconswitch vertex of a processor. The zeroth recon-switch vertex of a faulty processor is a unit-surplus source denoted s. Finally, there is an edge from each vertex corresponding to recon-switches linked to spares, to a sink vertex $t$. All edges have unit capacity. Note that, since the edges between recon-switch vertices of a processor have unit capacity, the subgraph corresponding to a processor models the contention for $v$-links when reconfiguration paths bend, as pointed out in Section 3.

Any flow path from a source to sink in $G$ obtained by a maxflow algorithm (e.g., in Fig. 8b) has a direct correspondence with a reconfiguration path from a fault to a spare in the actual hardware (e.g., in Fig. 8a). It is clear that a fault set $F$ will be reconfigurable if and only if there is a flow of value $|F|$ in $G$. We first used the $O\left(|V|^{3}\right)$ lift-to-front preflow-push algorithm of [9] to determine if a matching exists between the set of faults (of size $|F|$ ) and the set of associ- 


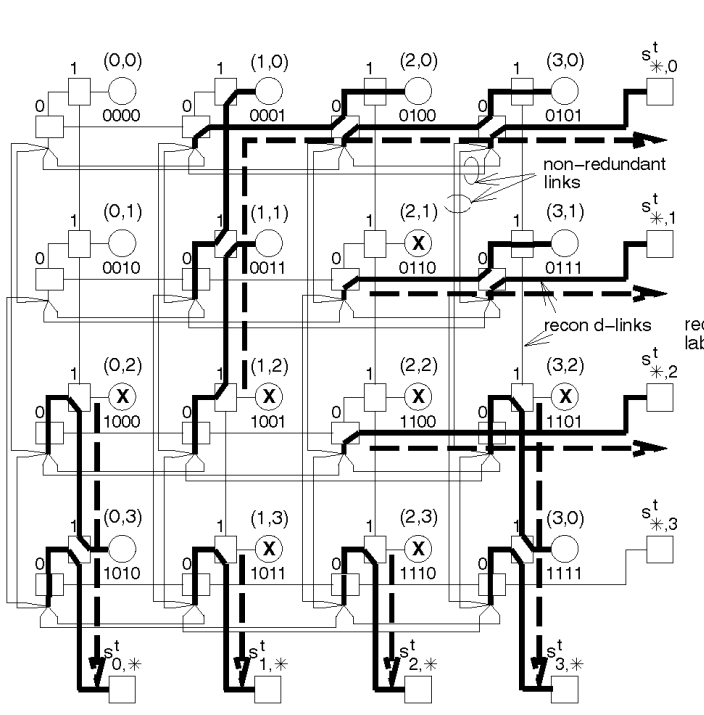

(a)

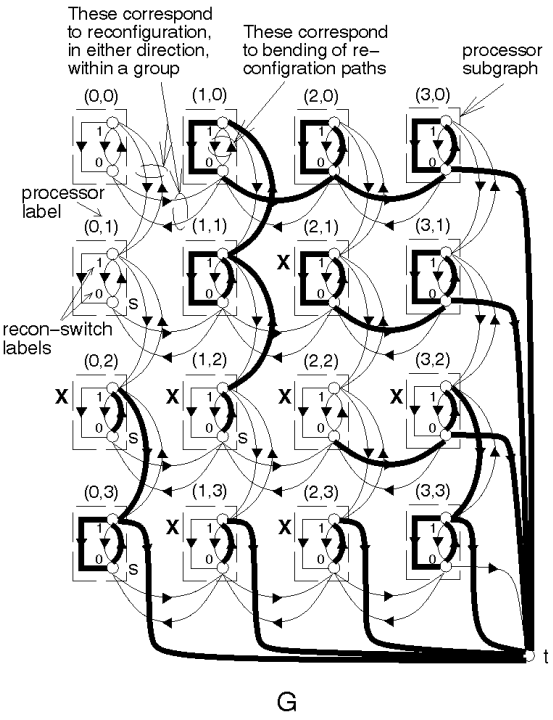

(b)

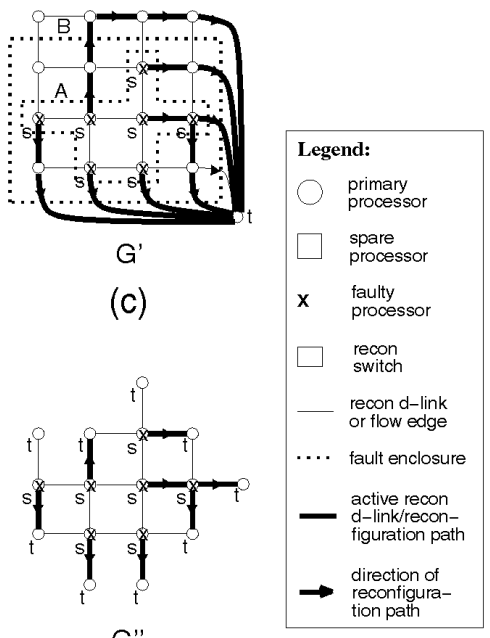

(d)

Fig. 8. (a) A four-dimensional FT hypercube based on the 2D-parity code showing settings of recon-switches determined by the reconfiguration algorithm of Section 6 to tolerate a fault pattern. (b) The flow graph $G$ corresponding to (a), along with the reconfiguration paths used for the different faults. (c) The simpler graph $G$ ' derived from $G$, showing fault enclosures $A$ and $B$ both of capacity 14 . (d) The flow graph $G$ ' used in determining DFT capabilities corresponding to the fault enclosure $A$ in $G$.

ated spares (of size $O(g)$ )-this takes $O\left((|F|+g)^{3}\right)$ time. $^{5}$ Only if this failed did we solve the maxflow problem associated with $G$ using the above algorithm in $O\left(N^{3}\right)$ time. For moderate number of faults (e.g., for $|F|<=40$ for a 1,024processor 2D-parity code based FT array using $g=64$ spares), a matching exists in a substantial number of cases ( $\geq 75$ percent of the cases in the above example), so that the reconfiguration time is reduced appreciably.

\section{Deterministic Fault Tolerance (DFT) CAPABILITIES}

\subsection{Preliminaries}

In this section, we derive the DFT capability, defined as the maximum number of faults guaranteed reconfigurable, for FT multiprocessors, based on the various codes, by determining a tolerable worst-case fault pattern of maximum size-we call these DFT fault patterns. For a given fault size, the worst-case fault pattern will be clustered rather than dispersed, since, in the former case, the capacity of a minimum source-sink cut ${ }^{6}$ in $G$ is no more than in the latter case, and, from the well-known maxflow-mincut theorem, this capacity gives the amount of flow possible, i.e., it gives the number of tolerable faults. We will be computing DFT capabilities at the corner and interior of the various FT layouts as follows: For the single-spare-per-group design, the corner DFT capability is computed at the corner of the lay-

5. One could also use the $O(\sqrt{|V|}|E|)$-time bipartite matching algorithm of [20], which, since there are $O(g)$ edges in the bipartite graph, would run in $O\left((|F|+g)^{1.5}\right)$ time.

6. A source-sink cut $(S, T)$ in $G=(V, E)$ is a partition of $V$ into $S$ and $T=V$ $-S$, such that $s \in S$ and $t \in T$. The capacity of a cut $(S, T)$ is the total capacity of all edges from a vertex in $S$ to a vertex in $T$. out that is not adjacent to spares (e.g., location of processor $(0,0)$ in Fig. 6 and $(1,0)$ in Fig. 7a), which is the worst case; in the double-spare case, the particular corner is immaterial. Also, the interior DFT capability in the single-spare case is computed by considering the DFT fault pattern to be sufficiently away from nonspare boundaries of the layout so that reconfiguration paths coming out of the DFT fault pattern on sides facing these boundaries can bend to access spares on the other boundaries (as in the case of the reconfiguration path for processor $(1,2)$ in Fig. 8a); in the doublespare case, there is no such consideration, since there are spares on all sides. Note that the number of corner DFT fault patterns will be constant, since there are a constant number of corners, while the number of interior DFT fault patterns for both single- and double-spare cases will increase with $N$, since the region over which such fault patterns are considered grows with $N$. Therefore, the likelihood of an interior relative to a corner DFT fault pattern will increase likewise with $N$.

Next, to determine the DFT fault pattern, we consider the maximum flow in a simpler flow graph $G^{\prime \prime}$ derived in two steps from the flow graph $G$ of Section 6 as follows. First, a flow graph $G^{\prime}=\left(V^{\prime}, E^{\prime}\right)$ is obtained from $G$ by replacing the processor subgraphs in $G$ by a single vertex as illustrated in Fig. 8c. We also replace the two oppositely directed edges between recon-switch vertices of adjacent processors in $G$ by a single undirected edge in $G^{\prime}$. Define a fault enclosure in $G^{\prime}$ as a minimal cut-set ${ }^{7}$ whose removal disconnects vertex sets $V_{1}$ and $V_{2}$, where $V_{1} \supseteq F$ and $V_{2}=V^{\prime}$ $-V_{1}$, and its capacity as the number of edges, called enclosure edges, it includes (see Fig. 8c). The graph $G^{\prime \prime}=\left(V^{\prime \prime}, E^{\prime \prime}\right)$ corresponding to a fault enclosure is that induced by the vertex set $V_{1}$ in $G^{\prime}$. In $G^{\prime \prime}$, every vertex on the fault enclosure

7. A minimal cut-set is a minimal set of edges whose removal destroys all paths between two mutually exclusive sets of vertices in some graph. 
is connected to a sink (see Fig. 8d). We will find reconfiguration paths in this simpler graph $G^{\prime \prime}$ to determine DFT capabilities using maxflow and matching concepts. From the above discussion, it is clear that, if $F$ is reconfigurable in $G$ (i.e., if $F$ is tolerable), then it will be so in $G^{\prime \prime}$ also. Generally, however, the converse is not necessarily true when there are bent reconfiguration paths in $G^{\prime \prime}$, since processor subgraphs in $G$, which modeled v-link contention during bending (as pointed out in Section 3) have been replaced by single vertices in $G^{\prime \prime}$. As we will see shortly, all reconfiguration paths in $G^{\prime \prime}$ in our DFT proofs are straight, and, thus, they are valid reconfiguration paths in $G$ that are hardware supportable. Finally, since boundary processor vertices connected to sinks in $G^{\prime \prime}$ may correspond to vertices of interior processors not adjacent to the sink (spares) in $G$ (e.g., processor $(1,1)$ in Figs. 8b and 8d), we need to ensure that a reconfiguration path formed until such an interior processor in $G$ can be extended from it to the sink (spare) in G. Below, we identify the DFT fault pattern for the 2D-parity code by finding flows for the fault pattern in $G^{\prime \prime}$ that meet this requirement, and, thereby, determine its DFT capability. For the other codes, we only state their DFT capabilities; derivations are similar to that for the 2D-parity code and can be found in [16].

\subsection{DFT Capabilities Associated with Various ECC Codes}

THEOREM 13. The corner DFT capability for the FT design based on the 2D-parity code is $\min (s, 5)$ when there is only one spare per processor group and $\min (s, 10)$ when there are two spares per processor group, and the interior DFT capability is $\min (s, 18)$, where $s$ is the number of spares.

PROOF. For the 2D-parity code, the least capacity of any enclosure for a fault pattern is equal to the perimeter of the circumscribing rectangle (see, e.g., Fig. 8c). Since of all rectangles of the same size, the square has the minimum perimeter, the worst-case fault pattern $F$ for any fault size will be a rectangle $(a, b, c, g)$ as close to a square as possible plus, possibly, a partial column $(e$, f) adjacent to it as in Fig. 9a, because, then, the fault pattern will be most difficult to tolerate. Specifically, $|F|$ will be either of the form $\left|F_{1}\right|=r^{2}+r^{\prime}$, where $0<$ $r^{\prime} \leq r$, or of the form $\left|F_{2}\right|=r(r+1)+r^{\prime}$, where $0<r^{\prime} \leq(r$ $+1)$, and, therefore, will correspond to an $r^{\prime}$-length column adjacent to either an $r \times r$ square or an $r \times(r+$ 1) rectangle, respectively. To determine the corner DFT capability, first assume one spare per processor group and consider the worst-case fault pattern of Fig. $9 \mathrm{a}$ to be in the left-top corner of the layout so that point $a$ is at the location of processor $(0,0)$ in Fig. 6 . Since there are no enclosure edges on sides $(a, b)$ and $(a, f)$ (because there are no edges leading to spares on those sides), the minimum capacity of any fault enclosure will be either $C_{1}=2 r+1$ or $C_{2}=2 r+2$, corresponding to $\left|F_{1}\right|$ and $\left|F_{2}\right|$ faults, respectively. Note that both $\left|F_{i}\right|$ and $C_{i}$ depend on just variables $r$ and $r^{\prime}$. Stepping through values $0,1,2, \ldots$ of $r$, and, for each such value, stepping through values 1 through $r$ (for $\left|F_{1}\right|$ faults) or $(r+1)$ (for $\left|F_{2}\right|$ faults) of $r^{\prime}$, we find that the largest fault size $\left|F_{i}\right|$, which does not exceed capacity $C_{i}, i=1,2$, occurs for $i=1$ for $\left|F_{1}\right|=C_{1}=5$ or for $r=2$ and $r^{\prime}=1$; denote this fault pattern by $F_{1}^{\max }$.

Note that, since $F_{1}^{\max }$ is the limiting fault pattern beyond which $|F|$ will exceed $C$, we have also established that, for any $F \subseteq F_{1}^{\max }$, the capacity (or perimeter) of its circumscribing rectangular enclosure will be at least $|F|$. If we now match faults to perimeter points of the circumscribing rectangle of $F_{1}^{\max }$, then, from the above result and Theorem 2, we have a matching, and, hence, from Theorem 3, there exist straight reconfiguration paths from each fault in $F_{1}^{\max }$ to a unique perimeter point on the circumscribing rectangle. These reconfiguration paths can be extended further straight till a spare is reached to obtain complete reconfiguration paths for all faults in $F_{1}^{\max }$. Thus, the corner DFT capability in the single-spare case is five or $s$, whichever is less. In the double-spare case, it is easy to see that the DFT fault pattern will be the same as in the single-spare case, but, with the five spares adjacent to sides $(a, b)$ and $(a, f)$ in Fig. 9a also faulty, thus, giving a DFT capability of $\min (s, 10)$.

Finally, consider the fault pattern of Fig. 9a, located in the interior of the layout. In this case, the minimum capacity of any enclosure is either $C_{1}=2(2 r+1)$ or $C_{2}=$ $2(2 r+2)$, corresponding to $\left|F_{1}\right|$ and $\left|F_{2}\right|$ faults, respectively. Again, stepping through values of $r$ and $r^{\prime}$ as in the corner fault pattern case above, we find that the limiting fault pattern corresponds to $\left|F_{1}^{\max }\right|=18$ or to $r=4$ and $r^{\prime}=2$. Similar to the corner DFT fault pattern, there exists a matching-based reconfiguration path from every fault in $F_{1}^{\max }$ to a unique perimeter point on the circumscribing rectangle. In the doublespare case, these reconfiguration paths can be extended straight up to a spare as before. However, in the single-spare case, two sides of the circumscribing rectangle (sides $(a, b)$ and $(a, f)$ in Fig. 9a) will not have spares on their side. In this case, if the fault pattern is sufficiently in the interior, reconfiguration paths exiting out of such sides can bend to access spares on sides that have spares (as, for example, in the case of the reconfiguration path for faulty processor $(1,2)$ in Fig. $8 b$ ). Thus, as before, we can extend reconfiguration paths from perimeter points to spares even on sides not directly associated with spares. Hence, the interior DFT capability is $\min (s, 18)$.

THEOREM 14. The corner DFT capability for the FT design based on the full-two code is $\min (s, 4)$ when there is only one spare per processor group and $\min (s, 8)$ when there are two spares per processor group, and the interior DFT capability is $\min (s-l, 18)$ in the single-spare case and $\min (s, 18)$ in the double-spare case, where $s$ is the number of spares and $l$ the distance of the fault pattern from the right primaryprocessor boundary. 


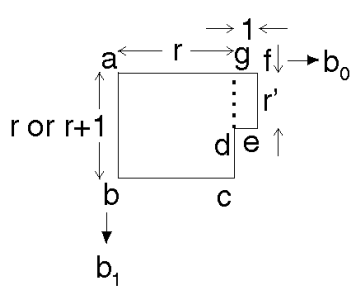

Fig. 9. Shape of worst-case fault pattern for the 2D-parity code.

THEOREM 15. The corner DFT capability for the FT design based on the 3D-parity code is $\min (s, 32)$ when there is only one spare per processor group and $\min (s, 64)$ when there are two spares per processor group, and the interior DFT capability is $\min (s, 234)$, where $s$ is the number of spares.

THEOREM 16. The corner DFT capability for the FT design based on the full-three code is $\min (s, 7)$ when there is only one spare per processor group and $\min (s, 11)$ when there are two spares per processor group, and the interior DFT capability is $\min \left(s, \frac{(s-l)(s-l+1)}{2}, 234\right)$ in the single-spare case and $\min (s, 234)$ in the double-spare case, where $s$ is the number of spares and $l$ the horizontal distance of the fault pattern from the right primary-processor boundary.

From the above results, it is evident that our probabilistic FT designs have good DFT capabilities, in addition to their high average-case fault tolerance which we will see in Section 8. Although the full-two and full-three codes based FT designs have smaller corner DFT capabilities than those based on the 2D- and 3D-parity codes, respectively, their interior DFT capabilities are equal (when $s$ is large enough, i.e., when $N$ is large enough, or when $l=0$ ). This is noteworthy, since the full-two and full-three codes based FT designs use a much smaller number of spares (in fact, they have the minimum number of spares of any degree-two and degree-three code based designs, respectively [18]) than the 2D- and 3D-parity code based designs, respectively.

\section{Reconfigurabilities and HaRdWARE Overheads of ECC-Based and Previous FT DESIGNS}

In this section, we compare our ECC-based probabilistic FT designs to some of the best deterministic [4], [6], [7], [8], [14], [27] and probabilistic [6], [27], [28] FT designs proposed previously, in terms of their reconfigurabilities and hardware overheads for two popular topologies, the 2D mesh, and the hypercube. In [6], [27], both deterministic and probablistic FT designs are presented; [6] gives several probabilistic designs, but we consider here only the design, denoted $M_{4}$, with the highest reconfigurability. Moreover, [14] gives two different deterministic designs, a globalsparing and a local-sparing design, that we will shortly discuss. The FT designs of [4], [7], [8], [14], like the ECC-based designs, are applicable to any topology, while the designs of [6], [27], [28] are specific to 2D meshes. ${ }^{8}$ First, in Section 8.1, we present reconfigurability results for the various ECC-

8. Actually, the design of [27] is applicable to constant dimensional toruses, but we consider only 2D meshes here. based FT designs and compare them to previous probabilistic designs employing the same number of spares. Then, in Section 8.2, we compare the hardware overheads of ECCbased FT designs to previous deterministic and probabilistic designs with similar MTTFs.

\subsection{Reconfigurability Comparisons}

We first summarize results on the reconfigurability of 1,024processor FT systems derived from various ECCs. The reconfigurability results were obtained using the maxflow-based reconfiguration algorithm of Section 6 and Monte Carlo simulations averaged over 1,000 samples for each fault size from $s$ (the number of spares) down to the largest fault size for which we get 100 percent reconfigurability-smaller fault sizes obviously have 100 percent reconfigurability. A 2D-parity code based FT system using 64 spares (single-spare case) was found to tolerate 92.8 percent of all 64-processor faults (the largest fault size tolerable), 98.8 percent of all 63-processor faults, and 100 percent of all smaller-sized faults; thus, $k_{\text {avg }}=63.93$. In the double-spare case, the same FT multiprocessor was able to tolerate 98.3 percent of all 128-processor faults and 100 percent of all smaller-sized faults $\left(k_{\text {avg }}=127.98\right)$. A full-two code based FT system using 46 spares (single-spare case) was able to tolerate 94.2 percent of all 46processor faults and 100 percent of all faults of smaller sizes $\left(k_{\text {avg }}\right.$ $=45.94$ ). In the double-spare case, this FT system was able to tolerate 100 percent of faults of sizes 92 (maximum possible) or smaller $\left(k_{a v g}=92\right)$. An FT system derived from the 3D-parity code had 100 percent reconfigurability for all fault sizes 363 (the total number of groups) and less $\left(k_{\text {avg }}=363\right)$; using two spares per group in this system is not practical-even one spare per group is quite expensive-hence, that case was not simulated. A full-three code based FT system had 100 percent reconfigurability in both the single and double spare cases (using 20 and 40 spares, respectively) for all fault sizes less than or equal to the number of spares ( $k_{\text {avg }}=20$ and 40 , respectively). Note that the average fault tolerance $k_{a v g}$ for the different ECC-based FT multiprocessors are approximately equal to the number of spares $s$, as desired.

As we saw in Section 5.2, FT systems derived from both the 2D-parity and full-two codes have reasonably low area overheads and are, therefore, cost-effective to build. Moreover, their DFT capabilities, derived in Section 7, and average-case fault tolerance, noted above, are quite high. The full-two code based system has the advantage that it has lower spare processor overhead and also, as seen above, is able to more effectively utilize its spares. The 2Dparity code based system, on the other hand, has smaller wiring area overhead (see Section 5.2). Therefore, the various codes considered here offer different trade-offs between wiring area/volume overheads, reconfiguration capability (or reliability/MTTF), and spare-processor overhead.

Next, in Fig. 10, we give reconfigurability results for the 2D-parity code based design, the probabilistic design $M_{4}$ of [6], and the three-track probabilistic design of [28], obtained using Monte Carlo simulations averaged over 1,000 samples. ${ }^{9}$ The various parameters of the probabilistic design of

9. The programs for ECC-based designs and that of [28] were coded by us, while that for [6] was provided by one of its authors, Dr. R. Cypher. 


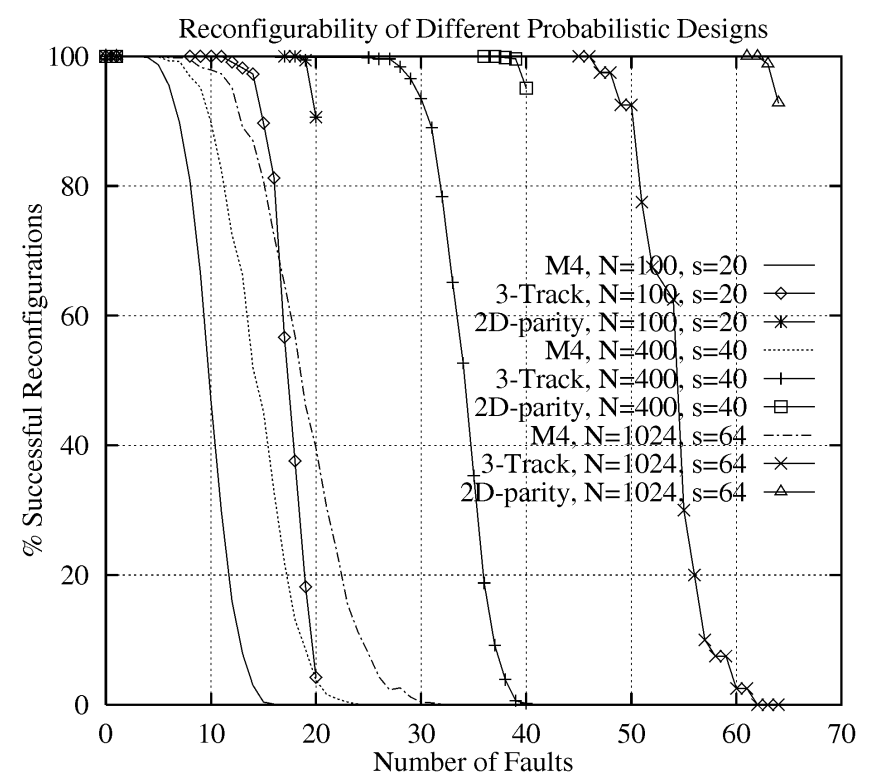

(a)

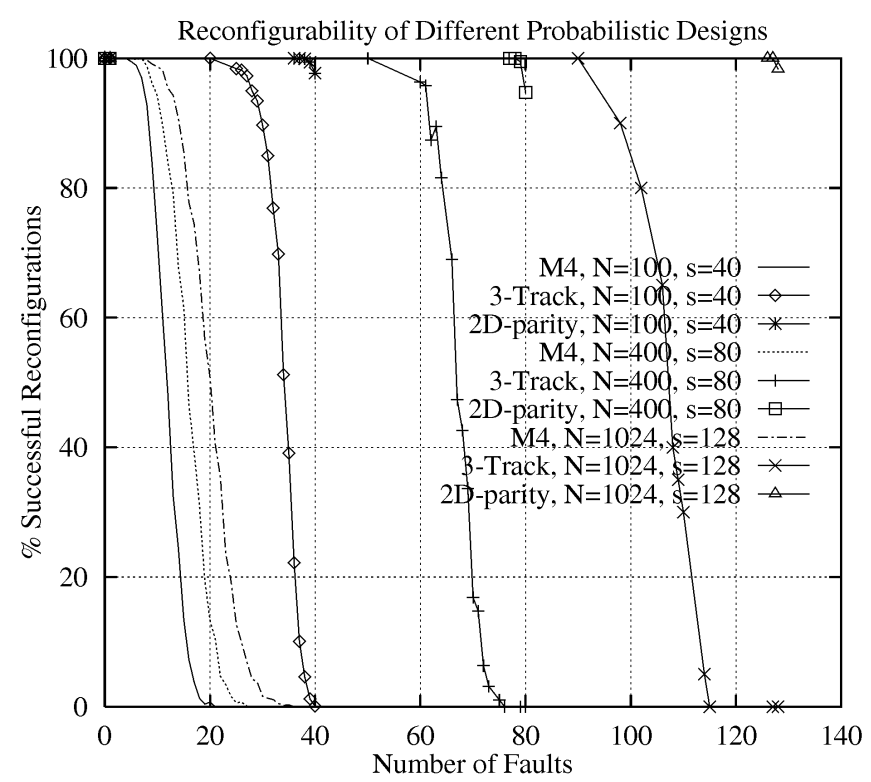

(b)

Fig. 10. Reconfigurability of three-track [28], $M_{4}$ [6], and 2D-parity code based probabilistic designs for arrays of $N=100,400$, and 1,024 processors for (a) $s=2 \sqrt{N}$ (single-spare case) and (b) $s=4 \sqrt{N}$ (double-spare case) spares.

[27] are given only in order terms (e.g., the number of spares is $\Theta(N))$ instead of in real terms, so that it could not be simulated to determine its reconfigurability; also, because of the constraints that different design parameters need to satisfy, the design of [27] appears to be applicable to only very large systems with at least hundreds of thousands of processors. Note also that the 2D-parity code based design (and, in general, any ECC-based design) is applicable to any topology, whereas the designs of [6], [28] are applicable to only 2D meshes. The figure shows plots of the percentage of successful reconfigurations averaged over 1,000 runs for fault sizes one through $s$ (the number of spares) for number of processors $N=100,400$, and 1,024, when the number of spares is kept the same-either $2 \sqrt{N}$ (single-spare case-Fig. 10a) or $4 \sqrt{N}$ (double-spare caseFig. 10b)-in all three designs. Note that the reconfigurability of our 2D-parity code based probabilistic design is 100 percent for all fault sizes except $s-2$ to $s$. Even for these large-sized faults, the reconfigurability is at least 90 percent and, in most cases, close to 100 percent. This is much better than the reconfigurability of the three-track design of [28], which, in turn, has much higher reconfigurability than the probabilistic design $M_{4}$ of [6] as seen from Fig. 10. These results also demonstrate that the spare-processor utilization in our probabilistic design is close-to-optimal, or, in other words, its average fault tolerance is close to the number $s$ of spares for different array sizes.

\subsection{Hardware Overhead Comparisons}

In Table 1, we compare the hardware overheads of the 2Dparity and full-two code based probabilistic FT designs to those of previous deterministic and probabilistic FT designs [7], [8], [4], [14], [13] with similar MTTFs. We assume that individual processor systems (including processor and as- sociated routing, memory, and other chips) have independent failure rates of $\lambda=0.011718$ failures/year and that their reliability functions follow the exponential failure law (i.e., $R$ $=1-e^{-\lambda t}$, where $R$ is the reliability and $t$ is the elapsed time). ${ }^{10}$ This corresponds to highly reliable single-processor subsystems, each with an MTTF of 85.34 years. As noted in the introduction, we are concerned here with multiprocessor systems in which it is important to preserve the original structure, in spite of any failures, for correct and nondegraded operation. For such a requirement, a single processor failure in a non-FT multiprocessor results in the failure of the entire system, i.e., the failure rate of an $N$-processor non-FT system is $N \lambda$. The MTTF of a non-FT 1,024-processor system, using processors with the above failure rate, is easily computed to be just one month. This dramatically underscores the need for fault tolerance in multiprocessor systems.

The hardware overheads (specifically, the redundant switch complexity and the redundant wire area) of an FT design depend upon the number of spares $s$. The hardware overheads given in Table 1 are those of designs with a number $s_{k}$ of spares such that their MTTFs are the same as those of a $k$-FT deterministic design. Before we compute other hardware overheads, we determine $s_{k}$ (last column in Table 1) for the different designs. First, consider the deterministic FT designs of [4], [6], [7], [8], [14], [27]. The designs of [4], [7], [8], [14] are $k$-FT deterministic global-sparing designs, i.e., they require $k$ spares to tolerate any $k$ or fewer faults, hence for them $s_{k}=k$. The designs of [6] and [27] are deterministic FT designs that require $s_{k}=2 k^{3}+4 k^{2}$ and $s_{k}=2 \sqrt{N} k^{4 / 3}+k^{8 / 3}$ spares, respectively, to be able to toler-

10. This failure rate for a processor and its associated hardware is reasonable considering that the failure rate of just a single Motorola $80 \mathrm{C} 386 \mathrm{mi}-$ croprocessor chip is 0.001752 failures/year [29]. 
TABLE 1

Comparison of the HaRdWARE OVERHEADS OF N-PROCESSOR FT 2D MESH AND HyPERCUBe SYSTEMS Based on the 2D-Parity AND FULL-Two Code Probabilistic Node-Covering MethodS to Those Based on Previous Deterministic and Probabilistic FT Methods

\begin{tabular}{|c|c|c|c|c|c|}
\hline FT Scheme & $\begin{array}{l}\text { Archi- } \\
\text { tecture }\end{array}$ & Degree & $\begin{array}{l}\text { Redundant } \\
\text { Switch } \\
\text { Complexity }\end{array}$ & $\begin{array}{l}\text { Redundant Wire Com- } \\
\text { plexity }\end{array}$ & $\begin{array}{l}\text { No. of } \\
\text { Spares } \\
\text { for } \\
\text { Equal } \\
\text { MTTF }\end{array}$ \\
\hline $\begin{array}{l}\text { Deterministic } \\
\text { Design (De- } \\
\text { coupling } \\
\mathrm{N} / \mathrm{Ws} \text { ) of [7] }\end{array}$ & $\begin{array}{l}\text { Hypercube } \\
\text { Mesh }\end{array}$ & $\begin{array}{l}\log N \\
4\end{array}$ & $\begin{array}{l}\Theta(N k \log N) \\
\Theta(4 N k)\end{array}$ & $\begin{array}{l}\Theta\left(\left((N+k)^{2} \log ^{2} N\right) / 2\right) \\
\Theta\left(8(N+k)^{2}\right)\end{array}$ & $\begin{array}{ll}k \\
k\end{array}$ \\
\hline $\begin{array}{l}\text { Deterministic } \\
\text { Design (Dio- } \\
\text { genes) of [ } 8]\end{array}$ & $\begin{array}{l}\text { Hypercube } \\
\text { Mesh }\end{array}$ & $\begin{array}{l}\log N \\
4 \\
\end{array}$ & $\begin{array}{l}\Theta(N(N+k)) \\
\Theta((N+k) \sqrt{N})\end{array}$ & $\begin{array}{l}\Theta(N(N+k) \log N) \\
\Theta(\sqrt{N}(N+k))\end{array}$ & $\begin{array}{l}k \\
k\end{array}$ \\
\hline $\begin{array}{l}\text { Deterministic } \\
\text { Design of [4] }\end{array}$ & $\begin{array}{l}\text { Hypercube } \\
\text { Mesh }\end{array}$ & $\begin{array}{l}\log N \\
4\end{array}$ & $\begin{array}{l}\Theta(N k \log N) \\
\Theta(2 N k)\end{array}$ & $\begin{array}{l}\text { Layout Not Given } \\
\text { Unknown }\end{array}$ & $\begin{array}{l}k \\
k\end{array}$ \\
\hline $\begin{array}{l}\text { Deterministic } \\
\text { Design of [6] }\end{array}$ & Mesh & 25 & $\begin{array}{l}\Omega\left(25\left(N+2 k^{3}+\right.\right. \\
\left.\left.4 k^{2}\right)\right)\end{array}$ & $\Theta\left(N k^{3}+2 k^{6}\right)$ & $2 k^{3}+4 k^{2}$ \\
\hline $\begin{array}{l}\text { Probabilistic } \\
\text { Design } M_{4} \text { of } \\
\text { [6] }\end{array}$ & Mesh & 8 & $\Omega(8(N+3.5580 k))$ & $\Theta(10 N)$ & $\lceil 3.5580 k\rceil$ \\
\hline $\begin{array}{l}\text { Deterministic } \\
\text { Design of [27] }\end{array}$ & Mesh & 8 & $\begin{array}{l}\Omega(8(N)+ \\
2 \sqrt{\left.\left.N k^{4 / 3}+k^{8 / 3}\right)\right)} \\
\end{array}$ & $\Theta\left(N k+2 \sqrt{N} k^{7 / 3}+k^{11 / 3}\right)$ & $\begin{array}{l}2 \sqrt{N} k^{4 / 3}+ \\
k^{8 / 3}\end{array}$ \\
\hline $\begin{array}{l}\text { Probabilistic } \\
\text { Design of }[27]\end{array}$ & Mesh & $\Theta\left(8 \log \log ^{2} N\right)$ & $\Omega\left(c_{2} N \log \log ^{2} N\right)^{a}$ & $\Theta\left(c_{1} N \log N \log \log ^{3.5} N\right)^{a}$ & Unknown \\
\hline $\begin{array}{l}\text { 3-Track } \\
\text { Probabilistic } \\
\text { Design of [28] }\end{array}$ & Mesh & 4 & $\Theta(9 N)$ & $\Theta(6 N)$ & $\lceil 1.2093 k\rceil$ \\
\hline $\begin{array}{l}\text { Deterministic } \\
\text { Node Cover- } \\
\text { ing with } \\
\text { Global Spar- } \\
\text { ing [14] }\end{array}$ & $\begin{array}{l}\text { Hypercube } \\
\text { Mesh }\end{array}$ & $\begin{array}{l}\log N \\
4\end{array}$ & $\begin{array}{l}\Theta(N k \log N) \\
\Theta(4 N k)\end{array}$ & $\begin{array}{l}\Theta\left(0.5 N^{3 / 2} k \log N\right) \\
\Theta(4 N k)\end{array}$ & $\begin{array}{l}k \\
k\end{array}$ \\
\hline $\begin{array}{l}\text { Deterministic } \\
\text { Node Cover- } \\
\text { ing with Lo- } \\
\text { cal Sparing } \\
{[14]}\end{array}$ & $\begin{array}{l}\text { Hypercube } \\
\text { Mesh }\end{array}$ & $\begin{array}{l}\log N \\
4\end{array}$ & $\begin{array}{l}\Theta(0.08 N k \log N) \\
\Theta(0.32 N k)\end{array}$ & $\begin{array}{l}\Theta\left(0.04 N^{3 / 2} k \log N\right) \\
\Theta(0.32 N k)\end{array}$ & $\begin{array}{l}\lceil 2.4837 k\rceil \\
\lceil 2.4837 k\rceil\end{array}$ \\
\hline $\begin{array}{l}\text { 2D-Parity } \\
\text { Code Based } \\
\text { Probabilistic } \\
\text { Node Cover- } \\
\text { ing }\end{array}$ & $\begin{array}{l}\text { Hypercube } \\
\text { Mesh }\end{array}$ & $\begin{array}{l}\log N \\
4\end{array}$ & $\begin{array}{l}\Theta(2 N \log N) \\
\Theta(8 N)\end{array}$ & $\begin{array}{l}\Theta\left(N^{3 / 2} \log N\right) \\
\Theta(8 N)\end{array}$ & $\begin{array}{l}\lceil 1.00139 k\rceil \\
\lceil 1.00139 k]\end{array} \mid$ \\
\hline $\begin{array}{l}\text { Full-2 } \\
\text { Code Based } \\
\text { Probabilistic } \\
\text { Node Cover- } \\
\text { ing }\end{array}$ & $\begin{array}{l}\text { Hypercube } \\
\text { Mesh }\end{array}$ & $\begin{array}{l}\log N \\
4\end{array}$ & $\begin{array}{l}\Theta(2 N \log N) \\
\Theta(8 N)\end{array}$ & $\begin{array}{l}\Theta\left(\sqrt{2} N^{3 / 2} \log N\right) \\
\Theta(\sqrt{2} \cdot 8 N)\end{array}$ & $\begin{array}{l}\lceil 1.00136 k\rceil \\
\lceil 1.00136 k\rceil\end{array} \mid$ \\
\hline
\end{tabular}

${ }^{a} c_{1}$ and $c_{2}$ are unknown constants.

All FT systems have the same MTTF as an N-processor k-FT deterministic system.

ate any $k$ or fewer faults. Finally, [14] also gives a deterministic local-sparing FT design of the type discussed in Section 3 , in which each group of $\sqrt{N}$ processors is $k_{1}$-FT with exactly $k_{1}$ spares per group, or $\sqrt{N} k_{1}$ spares in all. A 1,024processor deterministic local-sparing FT design with $k_{1}=5$ (or $\sqrt{1024} \times 5=160$ spares) is found to have an MTTF of 5.271 years, which lies between the MTTFs of deterministic global-sparing 64-FT (5.255 years) and 65-FT (5.333 years) designs. By linearly interpolating between the latter two MTTFs, we find that a 1,024-processor global-sparing FT design with $\left\lfloor 64+\frac{5.271-5.255}{5.333-5.255}\right\rfloor=\lfloor 64.42\rfloor$ spares will have, at most, the same MTTF as the above local-sparing design with 160 spares. Assuming that the above relationship between the number of spares in a global-sparing design and that in a local-sparing design with similar MTTF holds for all $\mathrm{N}$-processor systems, we conclude that a local-sparing design with $s_{k}=\left\lceil\frac{160}{64.42} \cdot k\right\rceil=\lceil 2.4837 k\rceil$ spares will have at least the same MTTF as a $k$-FT deterministic design. We found the $s_{k}$ values derived from the above assumption to be reasonably accurate for different $\mathrm{N}$-processor systems. For example, for $N=256$, the MTTF of a 32-FT globalsparing design is approximately 10.3 years compared to an MTTF of approximately 11.5 years for a local-sparing design with $\lceil 2.4837 \times 32\rceil=80$ spares, and, for $N=2,500$, the MTTF of a 100-FT global-sparing design is approximately 3.4 years compared to an MTTF of approximately 3.1 years for a local-sparing design with $\lceil 2.4837 \times 100\rceil \approx 250$ spares.

Next, we consider the probabilistic designs of [6], [28] 
and our ECC-based designs; the probabilistic design of [27], as pointed out in Section 8.1, could not be simulated to determine its reconfigurability/MTTF. To determine the $s_{k} \mathrm{~s}$ for these designs, we proceed in a manner similar to the case of local-sparing designs above. Thus, we find that the MTTF $M$ for 1,024-processor systems with $s$ spares of the probabilistic designs of [6], [28] and those based on the 2Dparity and full-two codes lies between the MTTFs $M^{\prime}$ and $M^{\prime \prime}$ of $k^{\prime}$-FT and $\left(k^{\prime}+1\right)$-FT deterministic designs, respectively, as follows.

1) For an $M_{4}$ design of [6] with $s=2 \sqrt{N}=64$ spares, $M=1.569$ years, $k^{\prime}=17, M^{\prime}=1.488$ years, and $M^{\prime \prime}=$ 1.570 years;

2) For a three-track design of [28] with $s=2 \sqrt{N}=64$ spares, $M=4.382$ years, $k^{\prime}=52, M^{\prime}=4.308$ years, and $M^{\prime \prime}=4.388$ years;

3) For a 2D-parity code based design with $s=2 \sqrt{N}=64$ spares, $M=5.248$ years, $k^{\prime}=63, M^{\prime}=5.176$ years, and $M^{\prime \prime}=5.255$ years;

4) For a full-two code based design with $s=\lceil\sqrt{2 N}\rceil=46$ spares, $M=3.827$ years, $k^{\prime}=45, M^{\prime}=3.752$ years, and $M^{\prime \prime}=3.832$ years.

From the above observations, we conclude that a probabilistic design with $s_{k}=\left\lceil\frac{s}{k^{\prime}+\frac{M-M^{\prime}}{M^{\prime}-M^{\prime}}} \cdot k\right\rceil$ spares will have at least the same MTTF as a $k$-FT deterministic design. Thus, we obtain:

1) For an $M_{4}$ design, $s_{k}=\lceil 3.5580 k\rceil$;

2) For a three-track design, $s_{k}=\lceil 1.2093 k\rceil$;

3) For a 2D-parity code based design, $s_{k}=\lceil 1.00139 k\rceil$; and

4) For a full-2 code based design, $s_{k}=\lceil 1.00136 k\rceil$.

Note that a 2D-parity code based and other ECC-based designs with $\lceil 1.00139 k\rceil$ spares (or any number of spares between one and $g$ for single-spare designs and one and $2 g$ for double-spare designs), can be obtained by combining the "regular" processor groups into larger ones and associating one (or two for double-spare designs) spares with these groups.

Having obtained the spare-processor overheads $s_{k}$ for the different FT designs with similar MTTFs, we now turn to their wiring and switch overheads. The hardware overheads of all previous designs given in Table 1, except those of the deterministic and probabilistic FT 2D mesh designs of [6], [27], can be found in the respective papers (or is clear from the discussions therein) or in [14]. Discussion of layouts and overhead computation of the designs of [6], [27] can be found in [16]. Hardware overheads of all designs are summarized in Table 1.

It is apparent from Table 1 that, for reasonable-MTTF systems, the probabilistic designs, except that of [27], will incur much lower hardware overheads than deterministic designs, since the latter will require sufficiently large $k$ values to ensure the required reliability. Note that, among competing deterministic designs [4], [7], [8], [14], the globaland local-sparing node-covering designs [14] have the least overheads for a given MTTF, with the former incurring less spare overhead and the latter less wiring and switch overheads. Among probabilistic designs, the 2D-parity and fulltwo code based designs have much less overhead (including spare overhead) compared to previous designs with similar MTTFs, as shown in Table 1. The wiring and switch overheads for the 2D-parity code based probabilistic design are the same as that for the corresponding two-FT deterministic global-sparing node-covering design. Also, the 2D-parity code based design is better than the local-sparing nodecovering design when the disjoint groups in the latter need to be made more than two-FT to obtain reasonable MTTF values. Finally, the full-two code based design has a somewhat larger wiring overhead compared to the 2D-parity code based design, as noted in Section 5.2, but has a smaller spare overhead. Another attractive feature of our probabilistic designs is that they can provide increasing reliability just by increasing the number of spares from one to $g$ (or $2 g$ in a double-spare design), while their other hardware overheads remain constant.

To conclude this section, we now give a specific example comparing the hardware overheads of the two best previous designs that are applicable to arbitrary topologies, viz., the deterministic node-covering designs with global and local sparing [14], and our 2D-parity code based probabilistic design. For 1,024-processor mesh and hypercube systems with MTTFs of approximately five years, the wiringarea overheads $f_{o}$ s (computed using the same $\frac{d / 4}{\sqrt{a}}$ values as in Section 5.2) and spare-processor overheads $s_{0}$ s for the above designs are:

1) 64-FT deterministic node-covering with global sparing [14]: $f_{o}($ mesh $)=2384 \%, f_{o}$ (hypercube $)=237.5 \%$, and $s_{0}=6.25 \%$;

2) Deterministic node-covering with local sparing [14] in which each disjoint processor group is five-FT: $f_{o}$ (mesh) $=100 \%, f_{0}$ (hypercube $)=15.63 \%$, and $s_{0}=15.63 \%$; and

3) 2D-parity code based probabilistic node-covering (single-spare design): $f_{o}($ mesh $)=44 \%, f_{o}$ (hypercube $)=$ $6 \%$, and $s_{0}=6.25 \%$ (see Section 5.2).

From the above discussions, it is clear that our probabilistic FT designs provide significant reductions in hardware cost compared to previous designs for comparable reliabilities/MTTFs.

\section{Conclusions}

Our aim was to design FT multiprocessors of any topology with low spare-link and switch overheads, but with very high average fault tolerance. We developed a methodology to effectively use linear ECCs to design such FT multiprocessors by partitioning them into intersecting FT processor groups based on the parity groups of an ECC. We showed that the deterministic error detectability and the average erasure correctability of linear codes are lower bounds for the deterministic and average fault tolerance, respectively, of the FT multiprocessor derived from them in this manner. These lower-bound results provide us with the rationale for using ECCs with high average erasure correctability, but low group degree, group size, and check-bit overhead to 
design FT multiprocessors-the latter metrics affect its hardware overhead. Such ECCs were identified in [18] to be the 2D-parity, 3D-parity, full-two, and full-three codes, and were used to design redundant disk arrays or RAIDs. We used these ECCs to design FT multiprocessors and developed efficient layout strategies for them. Our results show that, indeed, the resulting designs have very high average fault tolerance $\left(k_{\text {avg }} \approx s\right.$ ) or reliability with very small (six to nine percent) to reasonable (44-65 percent) area/volume overheads. Another attractive feature of our designs is that the processor degree in the FT system is the same as that in the non-FT system. We also showed that the deterministic fault tolerances of these designs are quite high. The above codes also offer various trade-offs between spare processor overhead, layout area or wiring overhead, and average fault tolerance, as specified earlier. The code whose tradeoffs match the cost constraints and reliability goals of the target system can be used to design the FT multiprocessor. Finally, we compared our probabilistic FT designs to some of the best deterministic and probabilistic designs proposed in the past, and showed that our designs can meet a given mean-time-to-failure (MTTF) specification at much lower hardware costs (switch complexity, redundant wiring area, and spare-processor overhead) than previous designs. Further, for a given number of spare processors, our designs were shown to have close-to-optimal reconfigurabilities that are much better than those of previous probabilistic designs. In conclusion, we believe that the methodology presented here offers a very cost-effective technique to build highly-available and highly-reliable multiprocessor systems of any topology that can be used in environments ranging from misson- or life-critical systems to business transaction processing.

\section{ACKNOWLEDGMENTS}

We thank Dr. R. Cypher for providing us the code to simulate the probabilistic FT mesh design $M_{4}$ of [6]. We also thank the referees for their helpful comments. This research was supported by U.S. National Science Foundation grant MIP-9210049.

\section{REFERENCES}

[1] B. Arazi, A Commonsense Approach to the Theory of Error-correcting Codes. MIT Press, 1988.

[2] P. Banerjee, S.Y. Kuo, and W.K. Fuchs, "Reconfigurable CubeConnected Cycles Architecture," Proc. 16th Fault Tolerant Computing Symp., pp. 286-291, June 1986.

[3] R.A. Brualdi, Introductory Combinatorics, pp. 155-158. New York: North Holland, 1977.

[4] J. Bruck, R. Cypher, and C.-T. Ho, "Wildcard Dimensions, Coding Theory and Fault-Tolerant Meshes and Hypercubes," Proc. 23rd Fault Tolerant Computing Symp., pp. 260-267, June 1993.

[5] J. Bruck, R. Cypher, and C.-T. Ho, "Fault-Tolerant Meshes and Hypercubes with Minimal Number of Spares," IEEE Trans. Computers, vol. 42, no. 9, pp. 1,089-1,104, Sept. 1993.

[6] J. Bruck, R. Cypher, and C.-T. Ho, "Fault-Tolerant Meshes with Small Degree," Proc. ACM Symp. Parallel Algorithms and Architectures, pp. 1-10, 1993.

[7] S.-C. Chau and A.L. Liestman, "A Proposal for a Fault-Tolerant Binary Hypercube," Proc. 19th Fault Tolerant Computing Symp., pp. 323330, Chicago, June 1989.

[8] F.R.K. Chung, F.T. Leighton, and A.L. Rosenberg, "Diogenes: A Methodology for Designing Fault-Tolerant VLSI Processor Ar- rays," Proc. 13th Fault Tolerant Computing Symp., pp. 26-31, June 1983.

[9] T.H. Cormen, C.E. Leiserson, and R.L. Rivest, Introduction to Algorithms. McGraw-Hill, 1990.

[10] S. Dutt and J.P. Hayes, "An Automorphic Approach to the Design of Fault-Tolerant Multiprocessors," Proc. 19th Fault Tolerant Computing Symp., pp. 496-503, Chicago, June 1989.

[11] S. Dutt and J.P. Hayes, "On Designing and Reconfiguring k-FaultTolerant Tree Architectures," IEEE Trans. Computers, vol. 39, no. 4, pp. 490-503, Apr. 1990.

[12] S. Dutt and J.P. Hayes, "Designing Fault-Tolerant Systems Using Automorphisms," J. Parallel and Distributed Computing, pp. 249268, July 1991.

[13] S. Dutt and J.P. Hayes, "A Local-Sparing Design Methodology for Fault-Tolerant Multiprocessors," to appear in the Special Issue on Graph Theory in Computer Science, Chemistry, and Other Fields of Computers and Mathematics with Applications, Elsevier Science.

[14] S. Dutt and J.P. Hayes, "Some Practical Issues in the Design of Fault-Tolerant Multiprocessors," IEEE Trans. Computers, vol. 41, no. 5, pp. 588-598, May 1992.

[15] S. Dutt, "Fast Polylog-Time Reconfiguration of Structurally FaultTolerant Multiprocessors," Proc. Fifth IEEE Symp. Parallel and Distributed Processing, pp. 762-770, Dec. 1993.

[16] S. Dutt and N.R. Mahapatra, "Node-covering, Error-correcting Codes and Multiprocessors with Very High Average Fault Tolerance," technical report, Univ. of Minnesota, Minneapolis, 1996accessible at ftp site: $\mathrm{ftp}$-mount.ee.umn.edu/pub/faculty/dutt/ftcomput/multiproc-ft/ieee-tc-rep96.ps.Z.

[17] S.H. Friedberg, A.J. Insel, and L.E. Spence, Linear Algebra. Englewood Cliffs, N.J.: Prentice Hall, 1979.

[18] G.A. Gibson, L. Hellerstein, R.M. Karp, R.H. Katz, and D.A. Patterson, "Failure Correction Techniques for Large Disk Arrays," Proc. ASPLOS '89, pp. 123-132, 1989.

[19] J.P. Hayes, "A Graph Model for Fault Tolerant Computing Systems," IEEE Trans. Computers, vol. 25, no. 9, pp. 875-883, Sept. 1976.

[20] J.E. Hopcroft and R.M. Karp, "An $n^{5 / 2}$ Algorithm for Maximum Matching in Bipartite Graphs," SIAM J. Computing, vol. 2, pp. 225231, 1973

[21] S.Y. Kung, VLSI Array Processors. Prentice Hall, 1988.

[22] F. Lombardi, M.G. Sami, and R. Stefanelli, "Reconfiguration of VLSI Arrays by Covering," IEEE Trans. Computers, vol. 8, no. 9, pp. 952-965, Sept. 1989.

[23] C.S. Raghavendra, A. Avizienis, and M.D. Ercegovac, "Fault Tolerance in Binary Tree Architectures," IEEE Trans. Computers, vol. 33, no. 6, pp. 568-572, June 1984.

[24] D.A. Rennels, "On Implementing Fault-Tolerance in Binary Hypercubes," Proc. 16th Fault Tolerant Computing Symp., pp. 344-349, June 1986.

[25] V.P. Roychowdhury, J. Bruck, and T. Kailath, "Efficient Algorithms for Reconfiguration in VLSI/WSI Arrays," IEEE Trans. Computers, vol. 39, no. 4, pp. 480-489, Apr. 1990.

[26] M. Sami and R. Stefanelli, "Reconfigurable Architectures for VLSI Processing Arrays," Proc. IEEE, vol. 74, no. 5, pp. 712-722, May 1986.

[27] H. Tamaki, "Construction of the Mesh and the Torus Tolerating a Large Number of Faults," Proc. ACM Symp. Parallel Algorithms and Architectures, pp. 268-277, 1994.

[28] T.A. Varvarigou, V.P. Roychowdhury, and T. Kailath, "Reconfiguring Processor Arrays Using Multiple-Track Models: The 3-Track-1Spare-Approach," IEEE Trans. Computers, vol. 42, no. 11, pp. 1,2811,293, Nov. 1993.

[29] L. Weil, M. Pecht, and E. Hakim, "Reliability Evaluation of Plastic Encapsulated Parts," IEEE Trans. Reliability, vol. 42, no. 4, pp. 536540, Dec. 1993. 


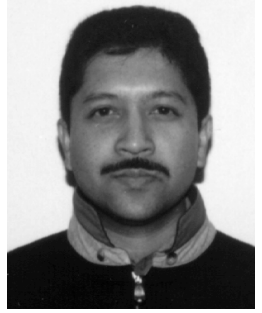

Shantanu Dutt (S'87-M'90) received the BE degree in electronics and communication engineering from the M.S. University of Baroda, India, in 1983, the MTech degree in computer engineering from the Indian Institute of Technology, Kharagpur, India, in 1984, and the PhD degree in computer science and engineering from the University of Michigan, Ann Arbor, in 1990.

In 1984-1985, he was a research and development engineer at CMC Ltd., Secunderabad, India. He is currently an associate professor in the Department of Electrical Engineering and Computer Science at the University of Illinois at Chicago. He was previously at the Department of Electricall Engineering, University of Minnesota, Twin Cities.

He was awarded a National Merit Scholarship by the Government of India, a University Fellowship by the M.S. University of Baroda, a Rackham Predoctoral Fellowship by the University of Michigan, and a Research Initiation Award by the U.S. National Science Foundation. His current technical interests include CAD for VLSI circuits, parallel and distributed computing, fault-tolerant computing, and computer architecture. He has published more than 30 papers journal and refereed conference papers in all these areas. He received a Best-Paper Award at the Design Automation Conference, 1996. On the occasion of the 25th anniversary of the Fault-Tolerant Computing Symposium (FTCS) in 1995, one of his papers (published in FTCS '88) in the area of fault-tolerant multicomputers was selected to be among the most influential papers published over the last 25 years in FTCS. He has been a session chair at the International Conference on Supercomputing, 1996, and at the scalability workshop at IEEE Parallel Processing Symposium, 1993. He is on the program committee of the Fault-Tolerant Computing Symposium, 1997/1998, and was on an U.S. National Science Foundation panel for selecting CAREER awards. He is a member of the IEEE, the IEEE Computer Society, and the ACM Special Interest Groups on Computer Architecture and Design Automation.

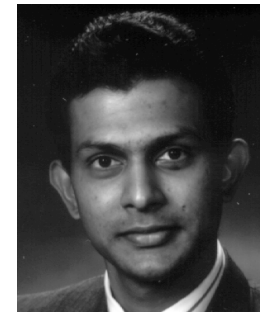

Nihar R. Mahapatra (S'91-M'96) received the BTech degree in electrical engineering from the Indian Institute of Technology, Delhi, India, in 1990, and the MS and PhD degrees in electrical engineering, both with a minor in computer and information science, from the University of Minnesota, Twin Cities, in 1993 and 1996, respectively. He is currently an assistant professor in the Department of Electrical and Computer Engineering at the State University of New York at Buffalo. His research interests include parallel and distributed processing, computer architecture and networking, VLSI, and fault-tolerance. He is on the program committee of the International Conference on Computer Design, 1997. He is a member of the IEEE Computer Society, and the ACM Special Interest Group on Computer Architecture. 